\title{
Isopentenyl diphosphate (IPP)-bypass mevalonate pathways for isopentenol production
}

Aram Kang ${ }^{1,2}$, Kevin W. George ${ }^{1,2}$, George Wang ${ }^{1,2}$, Edward Baidoo ${ }^{1,2}$, Jay D. Keasling ${ }^{1,2,3,4}$, Taek Soon Lee ${ }^{1,2, *}$

${ }^{1}$ Joint BioEnergy Institute, 5885 Hollis Street, Emeryville, CA 94608, USA.

${ }^{2}$ Biological Systems \& Engineering Division, Lawrence Berkeley National Laboratory, Berkeley, CA 94720, USA.

${ }^{3}$ Department of Bioengineering, University of California, Berkeley, CA 94720, USA.

${ }^{4}$ Department of Chemical and Biomolecular Engineering, University of California, Berkeley, CA 94720, USA.

${ }^{*}$ Corresponding author: Dr. Taek Soon Lee, Joint BioEnergy Institute, 5885 Hollis St. $4^{\text {th }}$ floor, Emeryville, CA 94608, USA; Phone: +1-510-495-2470, Fax: +1-510-495-2629, Email: tslee@lbl.gov

Keywords: Isopentenol, isoprenol, mevalonate pathway, biofuel, phosphomevalonate decarboxylase, IPP, toxicity, aeration 


\begin{abstract}
Branched $\mathrm{C}_{5}$ alcohols are promising biofuels with favorable combustion properties. A mevalonate (MVA)-based isoprenoid biosynthetic pathway for $\mathrm{C}_{5}$ alcohols was constructed in E. coli using genes from several organisms, and the pathway was optimized to achieve over $50 \%$ theoretical yield. Although the MVA pathway is energetically less efficient than the native methylerythritol 4-phosphate (MEP) pathway, implementing the MVA pathway in bacterial hosts such as E. coli is advantageous due to its lack of endogenous regulation. The MVA and MEP pathways intersect at isopentenyl diphosphate (IPP), the direct precursor to isoprenoid-derived $\mathrm{C}_{5}$ alcohols and initial precursor to longer chain terpenes, which makes independent regulation of the pathways difficult. In pursuit of the complete "decoupling" of the MVA pathway from native cellular regulation, we designed novel IPP-bypass MVA pathways for $\mathrm{C}_{5}$ alcohol production by utilizing promiscuous activities of two enzymes, phosphomevalonate decarboxylase (PMD) and an E. coli-endogenous phosphatase (AphA). These bypass pathways have reduced energetic requirements, are further decoupled from intrinsic regulation, and are free from IPP-related toxicity. In addition to these benefits, we demonstrate that reduced aeration rate has less impact on the bypass pathway than the original MVA pathway. Finally, we showed that performance of the bypass pathway was primarily determined by the activity of PMD. We designed PMD mutants with improved activity and demonstrated titer increases in the mutant strains. These modified pathways would be a good platform for industrial production of isopentenol and related chemicals such as isoprene.
\end{abstract}




\section{Introduction}

Isopentenol (3-methyl-3-buten-1-ol) is a potential biofuel and important precursor for flavor compounds (prenols and isoamyl alcohol esters) and industrial chemicals such as isoprene [1,2]. Two classes of metabolic pathways have been engineered to produce isopentenol in microbial hosts: amino acid production pathways utilizing 2-keto-acid intermediates $[3,4]$, and isoprenoid biosynthesis pathways, including both the mevalonate (MVA) [1,5-7] and non-mevalonate pathway (methylerythritol 4-phosphate (MEP) or 1deoxy-D-xylulose 5-phosphate (DXP) pathway) [8]. A heterologous MVA pathway was constructed to produce isopentenol in Escherichia coli by expressing 7 genes (Fig. 1Pathway O) [1]. To produce isopentenol, IPP is hydrolyzed by phosphatases such as NudF from Bacillus subtilis or NudB from E. coli. Although the initial performance of this pathway was low (8.3\% of pathway-dependent theoretical yield), subsequent optimization has significantly improved yields and titers [6,7]. Most recently, isopentenol was produced at a titer of $2.2 \mathrm{~g} / \mathrm{L}$ from $10 \mathrm{~g} / \mathrm{L}$ glucose, which is almost $70 \%$ of apparent theoretical yield [9].

A variety of engineering strategies have been applied to optimize the heterologous MVA pathway and improve isoprenoid production in E. coli [10-15]. In each case, balanced expression of pathway enzymes was required to maximize flux towards final products while minimizing the accumulation of toxic intermediates such as farnesyl diphosphate (FPP) [16], IPP [5-7,16], and 3-hydroxy-3-methyl-glutaryl-CoA (HMGCoA) [17]. For isopentenol production, the careful management of IPP levels is critical: engineering strategies to address its accumulation have included the deliberate "tuning" 
of the upstream MVA pathway [6] and the extensive overexpression of NudB, the phosphatase required to transform IPP into isopentenol [9].

Although the mechanism of IPP toxicity is unknown, the deleterious effects of its accumulation are clear. First, it has been demonstrated in various studies that accumulation of IPP inhibits cell growth $[5,6,16]$, which prevents a bioprocess from achieving enough cell biomass to maximize product titer. Even prior to affecting cell growth, it is likely that the transient accumulation of IPP induces a variety of stress responses as has previously been observed during the accumulation of FPP [14]. Responses to both generalized (e.g., RpoS-induced [18]) and condition-specific stress (e.g., acid stress [19], oxidative stress [20] and osmotic stress [21]) result in the recruitment of ATP-dependent defense mechanisms including DNA repair [19,20], ATPases [19], and ABC transporters [21]. The ATP cost of these processes may serve to compete with the energetically-expensive MVA pathway, reducing the yield and productivity of isoprenoid production. In the case of isopentenol production, high flux to IPP has an additional detrimental impact: through the action of $E$. coli native IPP isomerase (Idi), IPP can be diverted by native isoprenoid pathways that produce $\mathrm{C}_{10}$ - and $\mathrm{C}_{15}$-prenyl diphosphates (i.e. geranyl diphosphate (GPP) and FPP). The production of GPP and FPP decreases the carbon utilization efficiency of isopentenol production, and potentially inhibits MK activity, which in turn reduces MVA flux to the downstream enzyme reactions [22]. Moreover, isopentenol production via IPP requires the energetically expensive ATP-consuming formation of diphosphate prior to enzymatic hydrolysis. This diphosphate formation and subsequent hydrolysis is considerably inefficient in terms of atom and energy economy. Due to these factors, the "IPP- 
dependency" of the MVA pathway may intrinsically limit the engineering of the MVA pathway for more efficient isopentenol production.

In this work, we successfully decouple isopentenol production from IPP formation by constructing two novel "IPP-bypass" pathways. These two IPP-bypass pathways rely on decarboxylation of either MVA or MVA monophosphate (MVAP) for isopentenol production and do not produce IPP as an essential precursor for isopentenol. These optimized pathways eliminate the negative effects of IPP accumulation such as growth inhibition, energy-consuming stress responses, diverted carbon flux, and regulatory inhibition on mevalonate kinase (MK). We envision that these two IPPbypass pathways could open a new dimension of engineering the MVA pathway to produce isopentenol and isopentenol-derived valuable compounds such as isoprene.

\section{Materials and Methods}

\subsection{Strains and plasmid construction}

All strains and plasmids used in this study are listed in Table 1. Throughout the studies, E. coli BW25113 strain was used for isopentenol production, and E. coli $\mathrm{DH} 10 \mathrm{~B}$ was used for genetic cloning. The original sequence of $\mathrm{PMD}_{\mathrm{hv}}$ was obtained from NCBI database (HVO_1412, NC_013967.1), codon-optimized for expression in E. coli by GenScript (New Jersey, USA), and the optimized sequence was synthesized by IDT (Iowa, USA). A plasmid coding PMD $_{\text {se }}$ was received from Dr. Miziorko at University of Missouri [23], and the coding sequence was amplified by PCR for sub-cloning to expression vectors.

\subsection{Protein expression and purification}


A plasmid encoding a wild type mevalonate diphosphate decarboxylase from $S$. cerevisiae $\left(\mathrm{PMD}_{\mathrm{sc}}\right)$ with N-terminal His-tag $\left(\mathrm{pSKB} 3-\mathrm{PMD}_{\mathrm{sc}}\right)$ was transformed into $E$. coli BL21 (DE3). A seed culture of BL21 (DE3) harboring pSKB3-PMD sc $_{\text {was }}$ prepared by inoculating a single colony and growing it overnight in Luria-Bertani (LB) medium containing kanamycin $(50 \mu \mathrm{g} / \mathrm{mL})$. The seed culture was diluted in Terrific Broth supplemented with $2 \%$ glycerol and $50 \mu \mathrm{g} / \mathrm{mL}$ kanamycin and incubated at $37^{\circ} \mathrm{C}$ until the optical density of the culture at $600 \mathrm{~nm}\left(\mathrm{OD}_{600}\right)$ reached to $0.6-0.8$. The cell culture was supplemented with isopropyl- $\beta$-D-thiogalactopyranoside (IPTG) to the final concentration of $0.5 \mathrm{mM}$ and transferred to $18^{\circ} \mathrm{C}$ for protein expression overnight. Cells were collected by centrifugation and re-suspended in $50 \mathrm{mM}$ Tris- $\mathrm{HCl}(\mathrm{pH} \mathrm{7.5)}$ buffer containing $300 \mathrm{mM} \mathrm{NaCl}$ and $10 \mathrm{mM}$ imidazole. Cells were lysed by sonication and purified by HisPur Cobalt Resins (Thermo Scientific, USA). The purified PMD sc was desalted in $10 \mathrm{mM}$ Tris- $\mathrm{HCl}(\mathrm{pH} 7.5)$ containing $50 \mathrm{mM} \mathrm{NaCl}, 0.5 \mathrm{mM}$ dithiothreitol (DTT) and $20 \%$ glycerol, and flash-frozen in liquid nitrogen for storage at $-80^{\circ} \mathrm{C}$. All $\mathrm{PMD}_{\mathrm{sc}}$ mutants, $\mathrm{PMD}_{\text {se}}$, and NudB were purified as described above, except that NudB was desalted in $50 \mathrm{mM}$ Tris- $\mathrm{HCl}(\mathrm{pH} \mathrm{8.0)}$ buffer containing $0.1 \mathrm{mM}$ EDTA, $1 \mathrm{mM}$ DTT and $20 \%$ glycerol.

\subsection{Enzyme characterization and kinetics}

In vitro enzyme kinetics of decarboxylases were performed as described in previous studies $[23,24]$. Briefly, enzymatic activity of decarboxylase was determined by a spectrophotometer assay quantifying ADP product formation, which was coupled to NADH oxidation by pyruvate kinase/lactate dehydrogenase. Assay mixtures were prepared in $50 \mathrm{mM}$ HEPES-KOH (pH 7.5) containing $10 \mathrm{mM} \mathrm{MgCl}, 400 \mu \mathrm{M}$ 
phosphoenolpyruvate, $200 \mu \mathrm{M}$ NADH, $4 \mathrm{mM} \mathrm{ATP}$, and $25 \mathrm{U}$ of pyruvate kinase/lactate dehydrogenase (Sigma, P0294). The reaction was initiated by addition of various concentrations of MVAP from $100 \mu \mathrm{M}$ to $4,000 \mu \mathrm{M}$, and the reaction velocity was determined by monitoring OD at $340 \mathrm{~nm}$ in Spectramax 384plus microplate reader (Molecular Devices, USA).

\subsection{Isopentenol production in E. coli}

E. coli BW25113 harboring two plasmids was used for isopentenol production. Seed cultures of all production strains were prepared by growing single colonies in LB medium containing $100 \mu \mathrm{g} / \mathrm{mL}$ ampicillin and $30 \mu \mathrm{g} / \mathrm{mL}$ chloramphenicol overnight at $37^{\circ} \mathrm{C}$ with shaking at $200 \mathrm{rpm}$. The seed cultures were diluted in EZ-Rich defined medium (Teknova, USA) containing $10 \mathrm{~g} / \mathrm{L}$ glucose $(1 \%$, w/v), $100 \mu \mathrm{g} / \mathrm{mL}$ ampicillin and $30 \mu \mathrm{g} / \mathrm{mL}$ chloramphenicol. The E. coli cell cultures were incubated in rotary shakers $(200 \mathrm{rpm})$ at $37^{\circ} \mathrm{C}$, and $0.5 \mathrm{mM}$ IPTG was added to induce protein expression at $\mathrm{OD}_{600}$ of 0.6-0.8. To provide different levels of aeration, identical volumes of the cell culture were split into two flasks for incubation at $30^{\circ} \mathrm{C}$ with shaking at either $200 \mathrm{rpm}$ or $30 \mathrm{rpm}$.

For isopentenol quantification, $250 \mu \mathrm{L}$ of cell culture was combined with $250 \mu \mathrm{L}$ of ethyl acetate containing 1-butanol $(30 \mathrm{mg} / \mathrm{L})$ as an internal standard. This mixture of ethyl acetate and cell culture was vigorously shaken for $15 \mathrm{~min}$ and subsequently centrifuged at 13,000 g for 2 min to separate ethyl acetate from the aqueous phase. 100 $\mu \mathrm{L}$ of the ethyl acetate layer was diluted 5-fold, and $1 \mu \mathrm{L}$ was analyzed by Agilent GCMS equipped with Cyclosil-B column (Agilent, USA) or Thermo GCFID equipped with DB-WAX column (Agilent, USA) for quantitation of isopentenol. 


\subsection{Phosphatase screening}

To identify IP-hydrolyzing endogenous phosphatases, single gene knockout mutants of 36 phosphatases, of which substrates are mostly mono-phosphorylated metabolites, were retrieved from the Keio collection [25]. $1 \mathrm{~mL}$ overnight cultures from each mutant were concentrated in $0.5 \mathrm{~mL}$ of $50 \mathrm{mM}$ Tris- $\mathrm{HCl}(\mathrm{pH} 8.0)$ buffer containing $1 \mathrm{mM}$ DTT and $\sim 50 \mathrm{mg}$ of glass beads $(<100 \mu \mathrm{m}$, Sigma-Aldrich, USA). Cells were lysed by bead-beating for $2 \mathrm{~min}$ at $6.0 \mathrm{M} / \mathrm{s}$ (MP biomedicals Fast Prep, USA). After centrifugation of cell lysates at 20,000 g for $10 \mathrm{~min}$, clear supernatant was used for assay reaction containing $0.5 \mathrm{mM}$ isopentenyl monophosphate (IP). An equal volume of ethyl acetate was added to $100 \mu \mathrm{L}$ of assay reaction after incubating overnight at $30^{\circ} \mathrm{C}$, and isopentenol was extracted for $10 \mathrm{~min}$ by vigorous mixing.

Coding sequences of agp, aphA, and $y q a B$ were amplified from BW25113 genome by PCR, and they were subsequently cloned to pBbE1a vector [26] for overexpression. All primers used in this study are listed in Supplementary Table 1. Expression of these three genes were induced by addition of $0.5 \mathrm{mM}$ IPTG to the cell cultures, and cell lysates of each sample were prepared with three biological replicates as described above for screening of the 36 mutants. $600 \mu \mathrm{L}$ of assay reactions containing cell lysates, $1 \mathrm{mM}$ DTT were prepared and the reaction was initiated by addition of 0.5 $\mathrm{mM}$ IP. At each time point $(0,1,3,6$ and $22 \mathrm{hrs}), 100 \mu \mathrm{L}$ of the reaction mixture was sampled and combined with $100 \mu \mathrm{L}$ of ethyl acetate to extract isopentenol.

\subsection{Quantification of metabolites}

All metabolites were analyzed by liquid chromatography mass spectrometry (LCMS; Agilent Technologies 1200 Series HPLC system and Agilent Technologies 6210 
time-of-flight mass spectrometer) on a ZIC-HILIC column (150 mm length, 2.1-mm internal diameter, and 3.5- $\mu \mathrm{m}$ particle size). Standard chemicals (IPP and IP) were purchased from Sigma-Aldrich (USA). Metabolites were eluted isocratically with a mobile phase composition of $64 \%(\mathrm{v} / \mathrm{v})$ acetonitrile containing $50 \mathrm{mM}$ ammonium acetate with a flow rate of $0.15 \mathrm{~mL} / \mathrm{min}$. IPP and IP from E. coli extracts or enzyme assay were quantified via eight-point calibration curves ranging from $781.25 \mathrm{nM}$ to 200 $\mu \mathrm{M}$.

\section{Results and Discussions}

\subsection{Design rationale for IPP-bypass isopentenol pathways}

The biosynthesis of IPP from MVA consists of three energy-consuming reactions: two kinases (MK and phosphomevalonate kinase (PMK)) result in the formation of diphosphomevalonate (MVAPP), which is subsequently transformed by a decarboxylase (PMD) to form IPP. The diphosphate group of IPP is essential in chain elongation to produce GPP and FPP, and in the carbocation formation to produce cyclic terpenes since the removal of the diphosphate group is thermodynamically-favorable [27]. In isopentenol production via the MVA pathway, the alcohol is also produced by removal of the diphosphate group of IPP. However, this reaction is different from carbocation formation and does not require the diphosphate group as an essential leaving group to drive the hydrolysis reaction. Therefore, formation of the diphosphate group and its subsequent removal make the overall MVA pathway for isopentenol inefficient by unnecessarily consuming two ATPs.

To address the energetic limitations of IPP formation-and the deleterious effects of its accumulation - we designed two modified isopentenol pathways that bypass the 
formation of IPP (Fig. 1). The first modified pathway (pathway I) is designed for the direct conversion of MVA to isopentenol via ATP-driven decarboxylative elimination, and the second pathway (pathway II) is designed for a decarboxylative elimination of MVAP to IP followed by the hydrolysis of IP to isopentenol (Fig. 1). These modified pathways result in IPP-independent isopentenol production, which could relieve toxicity and prevent the loss of IPP flux to native pathways such as ubiquinone biosynthesis. Moreover, these two pathways reduce the complexity and energy cost of isopentenol production. As shown in Fig 1, direct decarboxylation of MVA (pathway I) reduces the number of enzymes required from 7 to 4 and the ATP requirement per molecule of isopentenol from 3 to 1 . In IPP-bypass pathway II, the number of enzymes is reduced from 7 to 5 and ATP molecules from 3 to 2 compared to the original pathway. Given the potential benefits of pathways I and II over the original MVA pathway (pathway O), we explored options to construct and express these optimized pathways in E. coli to produce isopentenol.

\subsection{Engineering of IPP-bypass pathway I and identification of promiscuous decarboxylase activity toward MVA and MVAP}

Engineering IPP-bypass pathways I and II requires a decarboxylase that converts MVA or MVAP to isopentenol or IP, respectively. Based on the chemical structures of the substrates and products (Fig. 1) and proposed mechanism of the decarboxylation reaction, we hypothesized that PMD might serve as a decarboxylase for MVA and MVAP in addition to its native substrate, MVAPP. Since PMD from S. cerevisiae $\left(\mathrm{PMD}_{\mathrm{sc}}\right)$ has been widely used for isoprenoid production in engineered $E$. coli $[6,7,28-$ 30], we initially chose $\mathrm{PMD}_{\mathrm{sc}}$ as the target PMD enzyme for each bypass pathway. 
$\mathrm{PMD}_{\text {sc }}$ was previously reported to convert 3-hydroxy-3-methylbutyrate (3-HMB) to isobutene [31], which supports the hypothesis that this enzyme has promiscuous decarboxylase activity.

With $\mathrm{PMD}_{\mathrm{sc}}$ as a potential decarboxylase for MVA, IPP-bypass pathway I was first constructed in E. coli by expressing three enzymes (AtoB, HMGS, and HMGR) to produce MVA along with $\mathrm{PMD}_{\mathrm{sc}}$ (strain ARK3a, Table 1). When the strain was tested in vivo, the engineered E. coli produced $0.85 \pm 0.18 \mathrm{mg} / \mathrm{L}$ isopentenol (Fig. 2B) while the control strain, which expressed only AtoB, HMGS, and HMGR without $\mathrm{PMD}_{\mathrm{sc}}$ (strain ARK3b, Table 1) did not show any detectable level of isopentenol (Fig. 2A). The fragmentation pattern and retention time of the isopentenol peak detected in strain ARK3a matched those from a 3-methyl-3-buten-1-ol standard (Fig. 2C). In vitro activity measurement was attempted to determine the kinetic parameters of $\mathrm{PMD}_{\mathrm{sc}}$ for MVA, but the enzyme activity was too low to determine the kinetic parameters (data not shown).

Structural analysis of a homologous PMD from Staphylococcus epidermis $\left(\mathrm{PMD}_{\text {se}}\right)$ [23] suggested that the diphosphate group is important for substrate binding even though it is not directly involved in the catalytic decarboxylation reaction (Supplementary Fig. S1). The importance of the diphosphate group in PMD $\mathrm{Pe}_{\text {se }}$ activity implies that the monophosphorylated substrate (i.e. MVAP) might be better suited for decarboxylation than the substrate without any phosphate group (i.e. MVA).

\subsection{Engineering of IPP-bypass pathway II and pathway optimization in E. coli}

To verify the improved activity of $\mathrm{PMD}_{\mathrm{sc}}$ for phosphorylated substrates (MVAP), an in vitro assay was performed with both MVA and MVAP. While isopentenol was not detected in the in vitro reaction, a detectable amount of IP was produced when MVAP 
was used as a substrate for $\mathrm{PMD}_{\text {sc. Th }}$ This result indicates that $\mathrm{PMD}_{\mathrm{sc}}$ has higher decarboxylase activity towards MVAP than MVA and suggests that the phosphate group of MVAP does indeed enhance substrate binding and catalysis (Supplementary Fig. S1). The $\mathrm{k}_{\mathrm{cat}}\left(0.14 \mathrm{~s}^{-1}\right)$ and $\mathrm{K}_{\mathrm{m}}(0.99 \mathrm{mM})$ of PMD $\mathrm{sc}_{\mathrm{sc}}$ toward MVAP (Supplementary Fig. S2), were about 35-fold lower and 8-fold higher than the reported $\mathrm{k}_{\mathrm{cat}}\left(4.9 \mathrm{sec}^{-1}\right)$ and $\mathrm{K}_{\mathrm{m}}(123$ $\mu \mathrm{M})$ toward the native substrate (MVAPP), respectively [32].

With a confirmation of promiscuous PMD $_{\text {sc }}$ activity for MVAP, we constructed a new IPP-bypass pathway (pathway II in Fig. 1) by expressing AtoB, HMGS, HMGR, MK, and $\mathrm{PMD}_{\mathrm{sc}}$ in E. coli (strain ARK2a, Table 1). Strain ARK2a produced $474.7 \mathrm{mg} / \mathrm{L}$ of isopentenol, a 558-fold improvement over the strain with pathway I (strain ARK3a). This new strain (strain ARK2a) achieved about $62.4 \%$ of the titer of the original isopentenol pathway (pathway $\mathrm{O}$ with strain ARK1a). It is noteworthy that IPP-bypass pathway II could produce isopentenol even without over-expressing any additional phosphatase that would hydrolyze the phosphate group in IP, which will be discussed in detail in the next section.

\subsection{Identification of endogenous phosphatase for IP}

The successful production of isopentenol via IPP-bypass pathway II suggested that endogenous $E$. coli phosphatases are capable of hydrolyzing IP to isopentenol. Initially, we hypothesized that IP might be hydrolyzed by promiscuous activities of Nudix hydrolases such as NudB in E. coli or an E. coli homolog of B. subtilis NudF, both of which were previously used to convert IPP to isopentenol $[1,5]$. In the original IPPdependent isopentenol pathway, the expression of NudB or NudF was essential for isopentenol production from IPP, and in vitro kinetic experiments of NudB showed that 
IPP was hydrolyzed by the enzyme [1]. However, the previous assay was based on the detection of the monophosphate formation without analyzing the final product by LCMS or GCMS, and it was not determined whether NudB hydrolyzes IPP by two consecutive hydrolysis reactions of two monophosphates or by a single hydrolysis reaction of a diphosphate group. We hypothesized that if NudB hydrolyzes IPP via the former fashion (i.e. two consecutive hydrolyses), both IP (intermediate) and isopentenol would be detected from an in vitro assay containing purified NudB and IPP. Interestingly, an in vitro assay of purified NudB with IPP produced only IP—no isopentenol was detected even after an extended incubation of 16 hours (Fig. 3A). Similarly, purified NudB could hydrolyze DMAPP to DMAP, but the final hydrolysis product, 3-methyl-2-butenol, was not detected (Supplementary Fig. S3A). In addition to NudB, NudF of B. subtilis, which was identified as a IPP hydrolase in a previous study [5], was also found to hydrolyze IPP to IP, but not to isopentenol (Supplementary Fig. S3B). On the other hand, it was confirmed in vitro that crude cell lysates of E. coli did hydrolyze IP to isopentenol (Fig. 3B). This result suggests that in the original isopentenol pathway, NudB hydrolyzed IPP to IP, but the following hydrolysis of IP to isopentenol was catalyzed by unknown endogenous phosphatase(s) in E. coli.

To identify the unknown endogenous phosphatase(s), phosphatase single gene knockout mutants were tested for their capability to hydrolyze IP to isopentenol. We reasoned IP hydrolysis to isopentenol would significantly decrease if the responsible IPhydrolyzing enzyme was absent in the knockout mutant strain. A total of 36 monophosphatase single gene knockout mutant strains were obtained from the Keio collection [25], and cell lysate from each individual strain was incubated with IP in vitro. 
Cell lysates from three single gene knockout mutant strains ( $\triangle a g p, \Delta a p h A$ and $\Delta y q a B$ ) produced significantly less isopentenol than the average level of isopentenol produced by all strains tested including the wild type (Fig. 4A). Even after 26 hours of incubation, the relative isopentenol level produced from cell lysates of $\triangle a g p, \triangle a p h A$ and $\triangle y q a B$ mutants were only $62 \%, 64 \%$ and $82 \%$ of the level from the wild type, respectively (Fig. 4B). It is noteworthy that cell lysates of all 36 mutants has some IP-hydrolyzing activity, suggesting that multiple endogenous phosphatases capable of hydrolyzing IP.

IP-hydrolysis efficiency significantly increased when one of these three genes, $a p h A$, was overexpressed both in wild type and in the $a p h A$-knockout mutant. In these strains, IP was completely converted into isopentenol immediately after addition of the IP to hydrolysates reactions (Fig. 4C). On the other hand, overexpression of the other two genes (agp and yqaB) showed relatively much slower IP hydrolysis rates (Fig. 4C), suggesting that $a p h A$ has much higher IP-hydrolysis activity than those of agp and $y q a B$. Co-expression of aphA along with pathway II (AtoB, HMGS, HMGR, MK and PMDsc; strain ARK2aa) resulted in an isopentenol titer of $705 \mathrm{mg} / \mathrm{L}$ after 31 hours of incubation, which is about $20 \%$ higher than that of the strain without aphA overexpression (strain ARK2a; Fig. 5) and $83 \%$ of the maximum titer of the original isopentenol pathway (pathway $\mathrm{O}$ in Fig. 1; strain ARK1a in Table 1; $836.9 \mathrm{mg} / \mathrm{L}$ ). Although AphA is a membrane-bound protein whose overexpression frequently is detrimental and inhibits growth [33], there was no significant growth difference between strains with or without aphA-overexpression (strains ARK2aa and ARK2a, respectively). Achieving a significant improvement in conversion of IP to isopentenol by aphA overexpression, we reasoned that the overall flux to isopentenol in strain ARK2aa could be further improved 
by increasing the activity of $\mathrm{PMD}_{\mathrm{sc}}$ toward MVAP. We thus focused on improving the promiscuous activity of $\mathrm{PMD}_{\mathrm{sc}}$ toward non-native substrates.

\subsection{PMD engineering for improved activity toward mevalonate monophosphate}

To engineer the active site of $\mathrm{PMD}_{\mathrm{sc}}$ for the non-native substrate MVAP, we first identified amino acid residues in PMD putatively responsible for binding the native substrate (MVAPP). Since the only X-ray crystal structure of $\mathrm{PMD}_{\mathrm{sc}}$ was solved without a bound substrate [34], the coordinates of MVAPP in the active site of PMDsc were predicted by aligning the crystal structure of $\mathrm{PMD}_{\mathrm{sc}}$ (PDB\#: 1FI4) to that of the homologous PMD enzyme from S. epidermis $\left(\mathrm{PMD}_{\text {se, }}\right.$ PDB\#: 4DPT) (Supplementary

Fig. S4A). Crystal structures of $\mathrm{PMD}_{\text {se }}$ were solved with two substrate analogs: adenosine 5-[ $[\gamma$-thio]triphosphate $(\mathrm{ATP} \gamma \mathrm{S})$ and 6-fluoromevalonate 5-diphosphate (FMVAPP) [23]. Alignment of $\mathrm{PMD}_{\mathrm{sc}}$ and $\mathrm{PMD}_{\text {se }}$ amino acid sequences showed 50\% similarity by BLAST search and revealed conserved residues for catalysis and substrate binding (Supplementary Fig. S4B). However, in vivo isopentenol production with IPPbypass pathway II and $\mathrm{PMD}_{\text {se }}$ (strain ARK4) was significantly reduced relative to $\mathrm{PMD}_{\mathrm{sc}}$ (11.3 $\mathrm{mg} / \mathrm{L}$ vs $474.7 \mathrm{mg} / \mathrm{L}$ after $24 \mathrm{hrs})$, suggesting that the activity of PMD $_{\text {se }}$ toward MVAP could be much lower than that of $\mathrm{PMD}_{\text {sc. }}$ The $\mathrm{k}_{\mathrm{cat}} / \mathrm{K}_{\mathrm{m}}$ ratios of $\mathrm{PMD}_{\mathrm{sc}}$ and $\mathrm{PMD}_{\mathrm{se}}$ are $4.0 \times 10^{4} \mathrm{~s}^{-1} \mathrm{M}^{-1}$ [32] and $6.5 \times 10^{5} \mathrm{~s}^{-1} \mathrm{M}^{-1}$ [23], respectively, which indicates higher substrate specificity of $\mathrm{PMD}_{\text {se }}$ for MVAPP. Increased substrate specificity in $\mathrm{PMD}_{\text {se }}$ could be attributed to the positively charged arginine at residue 193 (R193) [23]. R193 of $\mathrm{PMD}_{\mathrm{se}}$ is located within hydrogen bonding distance of the $\beta$-phosphate moiety of MVAPP and stabilizes the binding of MVAPP to the enzyme. On the other hand, $\mathrm{PMD}_{\mathrm{sc}}$ has a neutral threonine residue in the homologous position (T209) instead of the 
positively charged arginine (Supplementary Fig. S4B), and this perhaps allows the promiscuity of $\mathrm{PMD}_{\mathrm{sc}}$ towards the less negatively charged MVAP.

After we engineered the bypass pathway II with $\mathrm{PMD}_{\mathrm{sc}}$, an archaeal MVAPspecific decarboxylase was identified in Haloferax volcanii $\left(\mathrm{PMD}_{\mathrm{hv}}\right)$ with much better kinetics for MVAP $\left(\mathrm{K}_{\mathrm{m}}\right.$ of $0.159 \mathrm{mM}$ and $\mathrm{k}_{\mathrm{cat}}$ of $3.5 \mathrm{~s}^{-1}$ for MVAP; and no activity toward MVAPP) [24]. Unlike the conventional MVA pathway that supplies IPP via decarboxylation of MVAPP, the archaeal MVA pathway produces IPP via phosphorylation of IP, which is produced by decarboxylation reaction of MVAP similar to our bypass pathway II. Therefore, $\mathrm{PMD}_{\mathrm{hv}}$ was expected to be a natural decarboxylase that can convert MVAP to IP in the IPP-bypass pathway II. Surprisingly, however, no isopentenol production was detected when four pathway genes in the bypass pathway II (AtoB, HMGS, HMGR and MK) were expressed in vivo along with $\mathrm{PMD}_{\mathrm{hv}}$ (strain ARK5; data not shown). An ATP-NADH coupled assay was also performed in vitro to detect the activity of $\mathrm{PMD}_{\mathrm{hv}}$ toward MVAP, but no ATP hydrolysis activity was observed either. In the previous work where $\mathrm{PMD}_{\mathrm{hv}}$ kinetics were determined, $\mathrm{PMD}_{\mathrm{hv}}$ was overexpressed in its native host, $H$. volcanii, at $42{ }^{\circ} \mathrm{C}$ in salt-rich $\mathrm{Hv}$-YPC media (containing $144 \mathrm{~g}$ of $\mathrm{NaCl}, 21 \mathrm{~g}$ of $\mathrm{MgSO}_{4} \cdot 7 \mathrm{H}_{2} \mathrm{O}, 18 \mathrm{~g}$ of $\mathrm{MgCl}_{2} \bullet 6 \mathrm{H}_{2} \mathrm{O}$ and $4.2 \mathrm{~g}$ of $\mathrm{KCl}$ [24]. Given that optimal growth temperatures and salt concentration in media of $H$. volcanii are different from those for $E$. coli, $\mathrm{PMD}_{\mathrm{hv}}$ could have been expressed but inactive in E. coli. Nonetheless, it was noteworthy that four residues from $\mathrm{PMD}_{\mathrm{sc}}$ that interact with $\beta$-phosphates of MVAPP were missing in $\mathrm{PMD}_{\mathrm{hv}}$ between threonine 186 (T186) and glutamate 187 (E187) [24]. In other homologous PMD sequences from species with conventional MVA pathways, these missing residues are rich in serine and 
arginine, which facilitates interaction with the phosphoryl moieties of MVAPP and ATP. Therefore, analysis of residues near $\beta$-phosphate of MVAPP in three PMDs suggested that the activity of $\mathrm{PMD}_{\mathrm{sc}}$ toward MVAP could be improved by re-designing the local electrostatic environment around the $\beta$-phosphate of MVAPP.

Based on structural analysis of these three PMDs $\left(\mathrm{PMD}_{\mathrm{sc}}, \mathrm{PMD}_{\mathrm{se}}\right.$ and $\left.\mathrm{PMD}_{\mathrm{hv}}\right)$, four residues (K22, S155, S208 and T209) of $\mathrm{PMD}_{\text {sc }}$ adjacent to the $\beta$-phosphate of the MVAPP were selected for engineering (Supplementary Fig. S5). While the original substrate MVAPP has a net charge of -4 , two alternative substrates, MVAP and MVA, have a net charge of -2 and 0 , respectively. To compensate for this reduced negative charge, two serine residues (S155 and S208) were mutated to negatively charged glutamate (E), and the other two residues near the phosphate moiety (K22 and T209) were mutated to neutral methionine (M) and negatively charged aspartate (D), respectively. In addition, we constructed two more mutants, R74H and I145F (Fig. 6A), which were previously shown to increase activity of $\mathrm{PMD}_{\mathrm{sc}}$ in the similar decarboxylation reaction for 3-hydroxy-3-methylbutyrate (3-HMB) to produce isobutene [31]. In vitro assay reactions using cell lysates of two serine-to-glutamate mutations (S155E and S208E) did not produce detectible amount of product, which suggests that these two mutations significantly reduced the activity of $\mathrm{PMD}_{\mathrm{sc}}$ toward MVAP unlike the other mutants (data not shown). The $\mathrm{K} 22 \mathrm{M}$ mutation increased $\mathrm{K}_{\mathrm{m}}$ and decreased $\mathrm{k}_{\mathrm{cat}}$, but the kinetic parameters of the T209D mutant were similar to those of the wild type (Table 2, Fig. 6B). Interestingly, the specificity of PMD $\mathrm{Psc}_{\mathrm{sc}}$ toward MVAP $\left(\mathrm{k}_{\mathrm{ca}} / \mathrm{K}_{\mathrm{m}}\right)$ with $\mathrm{R} 74 \mathrm{H}$ or $\mathrm{I} 145 \mathrm{~F}$ mutation was $220 \%$ and $147 \%$ of that of wild type, respectively. Although R74 and I145 are located near the active site, it is unlikely that these residues 
interact directly with substrates: distances from the $\alpha$-phosphate group of MVAPP are $12.5 \AA$ and $15.0 \AA$ A respectively (Fig. 6A). Therefore, the improved activity of the R74H and I145F mutants toward MVAP and 3-HMB suggests that these two mutations changed the conformation of the active site to accommodate less negatively charged substrates. Although R74H and I145F increased activity for MVAP and 3-HMB, two mutants did not show detectible hydrolysis activity on MVA.

After identifying two mutations in PMD $_{\text {sc }}$ that improve activity toward MVAP, we prepared E. coli strains overexpressing four enzymes (AtoB, HMGS, HMGR, MK) along with one of three different PMD mutants including R74H (stain ARK2aM1), I145F (strain ARK2a $\mathrm{a}_{2}$ ), or the double mutant (strain ARK2am3) to see whether improved specificity for MVAP would increase isopentenol production in IPP-bypass pathway II. As shown in Fig. 6C, R74H (strain ARK2a $\mathrm{a}_{\mathrm{M} 1}$ ) resulted in significantly improved productivity $(20.4 \mathrm{mg} / \mathrm{L} / \mathrm{hr})$ over wild type $(15.9 \mathrm{mg} / \mathrm{L} / \mathrm{hr})$ through 30 hours of batch fermentation. The I145F mutation (strain ARK2a $\mathrm{a}_{\mathrm{M} 2}$ ), however, reduced isopentenol titer and productivity in vivo even though this mutation improved in vitro enzyme activity (Table 2). Interestingly, when these two mutations were combined (strain ARK 2am3), the titer and productivity were recovered to the comparable level to those of $\mathrm{R} 74 \mathrm{H}$, which suggests that $\mathrm{R} 74 \mathrm{H}$ mutation was dominant over the I145F mutation.

Successful identification of PMD mutants that improve or significantly reduce isopentenol titer and productivity supports the hypothesis that the promiscuous activity of PMD toward MVAP is the current bottleneck of the IPP-bypass pathway II. Given the huge engineering space to explore various mutations that can potentially improve the 
activity of PMD toward MVAP, this result provides a clear opportunity to improve IPPbypass pathway II for isopentenol production.

\subsection{Effect of MVA levels on isopentenol production in the IPP-bypass pathway II}

We successfully engineered IPP-bypass MVA pathways for isopentenol production and showed that pathway II could be improved by facilitating two limiting reactions: hydrolysis of IP and decarboxylation of MVAP to IP. Next, we targeted the "top" portion of the MVA pathway with engineering that would modulate pathway flux to MVA and tested how this variation affects isopentenol production in IPP-bypass pathway II. Previously, heterologous MVA pathways were constructed and tested with various combinations of HMGS and HMGR, and different pairs of HMGS-HMGR resulted in different levels of MVA and final isoprenoid titers [6,10,17,22]. The MVA level was reported to affect MK activity by substrate inhibition [22], and therefore, optimizing MVA flux has been one approach to improve titers of isoprenoid products.

To evaluate the effects of MVA concentration in IPP-bypass pathway II, we reconstructed the original and the modified pathways with four different pairs of HMGS and HMGR in the "top" portion of the pathway (Fig. 1): non-codon optimized original sequences from $S$. cerevisiae genes (MevTo), E. coli-codon optimized sequences of $S$. cerevisiae genes (MevTco), HMGS and HMGR of Staphylococcus aureus (MTSA), and those of Delftia acidovorans (MTDA).

In accordance with the previous reports, the original IPP-dependent isopentenol pathways showed different isopentenol titers depending on which pairs of HMGS and HMGR were used (Fig. 7). Analysis of intracellular metabolites confirmed that expression of different pairs of HMGS and HMGR indeed resulted in various 
intracellular MVA concentrations in strains with both pathway $\mathrm{O}$ and pathway II (Supplementary Fig. S6A). Intriguingly, isopentenol titers from the strains containing IPP-bypass pathway II did not change much when the pairs of HMGR and HMGS are changed (Fig. 7), and similar levels of IP were also observed in the strains with pathway II (Supplementary Fig. S6D). This "insensitivity" of the isopentenol titer to various "top" portions and a similar level of IP in pathway II strains suggests that the determining factor of isopentenol production in pathway II could be the PMD activity toward MVAP rather than upstream pathway efficiency.

In addition, metabolite analysis showed that strains with pathway $\mathrm{O}$ or pathway II accumulated significantly high levels of IPP or MVAP, respectively, regardless of intracellular MVA concentrations (Supplementary Fig. S6). Interestingly, MVAP was

accumulated to considerably higher concentrations than that of IPP (100 200 mM for MVAP vs 30 60 mM for IPP) without any significant toxicity, which is consistent with the previous report that MVAP is not inhibitory to cell growth [16].

\subsection{Relief of IPP-toxicity in the bypass pathway II}

Previous studies showed that the performance of the original MVA pathway was sensitive to MK expression levels: low MK expression resulted in attenuated flux to IPP and isopentenol, but high levels led IPP accumulation and resulted in growth inhibition [6,9]. Interestingly, growth was restored when NudB was overexpressed in IPPaccumulating strain to relieve IPP-toxicity. In the current study, we demonstrated that NudB could hydrolyze IPP to IP, but not further to isopentenol (Fig. 3), suggesting that IPP has more detrimental effects on growth than does IP. 
Since the bypass pathway II does not produce IPP, we hypothesized that the pathway would be insensitive to changes in MK expression and free from related toxicity. To compare growth and isopentenol production in the original and IPP-bypass pathway (pathway O and pathway II) under IPP- or IP-accumulating conditions, respectively, two modifications were made to the strains ARK1a and ARK2a (Supplementary Fig. S7). First, to achieve a moderate level of MK expression in the control strains, we removed the promoter previous added for MK overexpression in the medium copy plasmids JBEI12056 and JBEI-9310. With this engineering, MK was expressed at a moderate level as the forth enzyme in the operon containing three enzymes for the top portion of the MVA pathway, and it resulted strains ARK1e (harboring JBEI-6818 and JBEI-6833) and ARK2e (harboring JBEI-12051 and JBEI-9314). Second, to achieve very high MK expression level, an additional copy of MK was added to the high copy plasmids, JBEI6833 and JBEI-9314, resulting ARK1f and ARK2f, respectively (Supplementary Fig. S7). Confirming the previous results [6], balancing flux in the upstream pathway was critical for growth and isopentenol production (Fig. 8). Growth and isopentenol production of ARK1f was significantly reduced showing sensitivity to expression levels of MK, but strains with pathway II was insensitive to down-regulation (ARK2f) or upregulation (ARK2e) of MK, and free from burden of IPP accumulation (Fig. 8).

\subsection{The effect of limited aeration on isopentenol production via IPP-bypass pathway}

After characterizing the IPP-bypass pathway II in E. coli (strain ARK2a), we tested whether this pathway would have any advantage over the original pathway under ATP-limited conditions. In general, ATP is most efficiently supplied via oxidative phosphorylation with oxygen as a final electron acceptor. As a result, aeration has been 
an important operation in industrial-scale fermentation, especially when ATP-demanding isoprenoid biosynthetic pathways are exploited. However, the aeration cost is usually one of the largest portions (up to $26 \%$ ) of the overall utility cost, and the cost would be on the order of $\$ 60$ million per year in a plant that processes 2000 MT of dry biomass per day [35]. Moreover, oxygen mass transfer is limited in large-scale fermenters, and this potentially creates a local micro-aerobic or anaerobic environment during fermentation. Therefore, the development of fermentation processes with reduced aeration rates can significantly reduce production cost and improve process efficiency. With this goal in mind, we investigated the impact of reduced aeration on isopentenol production with pathways $\mathrm{O}$ and II, which require 3 ATPs and 2 ATPs, respectively, to produce one molecule of isopentenol. To provide different aeration rates, we prepared a $50-\mathrm{mL}$ cell culture with an $\mathrm{OD}_{600}$ of $0.6-0.7$, split into two $25-\mathrm{mL}$ cell cultures in 250 -mL flasks, and continued to incubate at $30^{\circ} \mathrm{C}$ for induction (0.5 mM IPTG) at two different shaking speeds (30 rpm and $200 \mathrm{rpm})$.

Fig. 9A shows that the isopentenol titer of pathway O (strain ARK1a) was more significantly affected when aeration was limited by lowering the shaking speed from 200 rpm to $30 \mathrm{rpm}$. With a reduced aeration, strain ARK1a produced only $22 \%$ of the initial titer at $200 \mathrm{rpm}$ after $16 \mathrm{hr}-$ fermentation (Fig. 9B). The bypass pathway II (strain ARK2a), however, produced $40 \%$ at $16 \mathrm{hrs}$ and up to $60 \%$ of the titers under the higher aeration conditions at $24 \mathrm{hrs}$. It is noteworthy that the $\mathrm{OD}_{600}$ of strain ARK1a was higher than that of strain ARK2a under poor aeration condition (at $30 \mathrm{rpm}$ ). A better growth but significantly less isopentenol production of strain ARK1a suggests that the heterologous MVA pathway may compete for ATP with other essential cellular processes related to the 
growth, and when ATP supply is limited (i.e. under poor aeration conditions), strain ARK1a might reduce the carbon flux to the MVA pathway to reduce the energy usage for this ATP-consuming heterologous pathway. The strain with pathway II (strain ARK2a), however, produced a similar or even higher level of isopentenol under limited aeration conditions after $16 \mathrm{hrs}$ or $24 \mathrm{hrs}$ of fermentation, respectively (Fig. 9A and Supplementary Fig. S8). This result also suggests that the bypass pathway II would be more robust when aeration is limited, and a reduced ATP demand in strain ARK2a is possibly beneficial to the strain under oxygen-limited conditions. Therefore, more economic production of isopentenol could be feasible via the ATP-saving IPP-bypass pathway II by reducing aeration costs for large scale fermentation.

\section{Conclusion}

Isopentenol is a potential gasoline alternative and a precursor of commodity chemicals such as isoprene. In this study, we reported our efforts to remove "IPPdependency" of the original MVA pathway and to overcome limitations intrinsic to IPP accumulation and "unnecessary" consumption of ATPs for isopentenol production. By implementing two previously unidentified activities of $\mathrm{PMD}_{\mathrm{sc}}$ and $\mathrm{AphA}$, we demonstrated that considerable isopentenol titers could be achieved without producing IPP via the pathway II.

The IPP-bypass pathway II was shown to be a robust alternative to the original pathway (pathway O) for isopentenol production. This modified pathway was insensitive to both MVA level and MK expression level, and reduced the engineering burden to balance the upstream MVA pathway and IPP toxicity. Most significantly, the IPP-bypass 
pathway II was more competitive when aeration was limited, which would significantly lower operational costs for aeration in a large scale fermentation.

Finally, in this report, we found that the promiscuous activity PMD is ratelimiting. The identification of PMD as the rate-limiting step in these bypass pathways provides clear engineering opportunities. Although we constructed a few PMD mutants with improved activity toward MVAP, more concerted efforts to engineer PMD promiscuity or identify homologous enzymes should yield additional increases in isopentenol yield and productivity. With further engineering, these bypass pathways will provide valuable platforms for the energetically-favored production of isopentenol, isoprene, and related $\mathrm{C}_{5}$ compounds.

\section{Acknowledgements}

We thank Dr. Miziorko at University of Missouri-Kansas City for providing a plasmid containing the $\mathrm{PMD}_{\text {se }}$ gene and Dr. Konda at JBEI for discussion on techno-economic impact of this work. This work was part of the DOE Joint BioEnergy Institute (http://www.jbei.org) supported by the U.S. Department of Energy, Office of Science, Office of Biological and Environmental Research, through contract DE-AC0205CH11231 between Lawrence Berkeley National Laboratory and the U.S. Department of Energy. The United States Government retains and the publisher, by accepting the article for publication, acknowledges that the United States Government retains a nonexclusive, paid-up, irrevocable, world-wide license to publish or reproduce the published form of this manuscript, or allow others to do so, for United States Government purposes. 


\section{References}

[1] H.H. Chou, J.D. Keasling, Synthetic pathway for production of five-carbon alcohols from isopentenyl diphosphate., Appl. Environ. Microbiol. 78 (2012) 7849-7855. doi:10.1128/AEM.01175-12.

[2] P.P. Peralta-Yahya, F. Zhang, S.B. del Cardayre, J.D. Keasling, Microbial engineering for the production of advanced biofuels., Nature. 488 (2012) 320-328. doi:10.1038/nature11478.

[3] M.R. Connor, J.C. Liao, Engineering of an Escherichia coli strain for the production of 3-methyl-1-butanol, Appl. Environ. Microbiol. 74 (2008) 57695775. doi:10.1128/AEM.00468-08.

[4] M.R. Connor, A.F. Cann, J.C. Liao, 3-Methyl-1-butanol production in Escherichia coli: Random mutagenesis and two-phase fermentation., Appl. Microbiol. Biotechnol. 86 (2010) 1155-1164. doi:10.1007/s00253-009-2401-1.

[5] S.T. Withers, S.S. Gottlieb, B. Lieu, J.D. Newman, J.D. Keasling, Identification of isopentenol biosynthetic genes from Bacillus subtilis by a screening method based on isoprenoid precursor toxicity., Appl. Environ. Microbiol. 73 (2007) 6277-6283. doi:10.1128/AEM.00861-07.

[6] K.W. George, A. Chen, A. Jain, T.S. Batth, E. Baidoo, G. Wang, et al., Correlation analysis of targeted proteins and metabolites to assess and engineer microbial isopentenol production, Biotechnol. Bioeng. 111 (2014) 1648-1658. doi:10.1002/bit.25226. 
[7] Y. Zheng, Q. Liu, L. Li, W. Qin, J. Yang, H. Zhang, et al., Metabolic engineering of Escherichia coli for high-specificity production of isoprenol and prenol as next generation of biofuels., Biotechnol. Biofuels. 6 (2013) 57. doi:10.1186/1754-68346-57.

[8] H. Liu, Y. Sun, K.R.M. Ramos, G.M. Nisola, K.N.G. Valdehuesa, W.K. Lee, et al., Combination of entner-doudoroff pathway with MEP increases isoprene production in engineered Escherichia coli., PLoS One. 8 (2013) e83290. doi:10.1371/journal.pone.0083290.

[9] K.W. George, M.G. Thompson, A. Kang, E. Baidoo, G. Wang, L.J.G. Chan, et al., Metabolic engineering for the high-yield production of isoprenoid-based C5 alcohols in E. coli, Sci. Rep. 5 (2015). doi:10.1038/srep11128.

[10] B.F. Pfleger, D.J. Pitera, C.D. Smolke, J.D. Keasling, Combinatorial engineering of intergenic regions in operons tunes expression of multiple genes., Nat. Biotechnol. 24 (2006) 1027-1032. doi:10.1038/nbt1226.

[11] J.R. Anthony, L.C. Anthony, F. Nowroozi, G. Kwon, J.D. Newman, J.D. Keasling, Optimization of the mevalonate-based isoprenoid biosynthetic pathway in Escherichia coli for production of the anti-malarial drug precursor amorpha-4,11diene, Metab Eng. 11 (2009) 13-19. doi:10.1016/j.ymben.2008.07.007.

[12] J.E. Dueber, G.C. Wu, G.R. Malmirchegini, T.S. Moon, C.J. Petzold, A.V. Ullal, et al., Synthetic protein scaffolds provide modular control over metabolic flux., Nat. Biotechnol. 27 (2009) 753-759. doi:10.1038/nbt.1557.

[13] A.M. Redding-Johanson, T.S. Batth, R. Chan, R. Krupa, H.L. Szmidt, P.D. Adams, et al., Targeted proteomics for metabolic pathway optimization: 
Application to terpene production., Metab. Eng. 13 (2011) 194-203. doi:10.1016/j.ymben.2010.12.005.

[14] R.H. Dahl, F. Zhang, J. Alonso-Gutierrez, E. Baidoo, T.S. Batth, A.M. ReddingJohanson, et al., Engineering dynamic pathway regulation using stress-response promoters., Nat. Biotechnol. 31 (2013) 1039-1046. doi:10.1038/nbt.2689.

[15] F. Zhu, X. Zhong, M. Hu, L. Lu, Z. Deng, T. Liu, In vitro reconstitution of mevalonate pathway and targeted engineering of farnesene overproduction in Escherichia coli, Biotechnol. Bioeng. 111 (2014) 1396-1405. doi:10.1002/bit.25198.

[16] V.J.J. Martin, D.J. Pitera, S.T. Withers, J.D. Newman, J.D. Keasling, Engineering a mevalonate pathway in Escherichia coli for production of terpenoids., Nat. Biotechnol. 21 (2003) 796-802. doi:10.1038/nbt833.

[17] D.J. Pitera, C.J. Paddon, J.D. Newman, J.D. Keasling, Balancing a heterologous mevalonate pathway for improved isoprenoid production in Escherichia coli, Metab. Eng. 9 (2007) 193-207. doi:10.1016/j.ymben.2006.11.002.

[18] R. Hengge, The two-component network and the general stress sigma factor RpoS (sigma S) in Escherichia coli., Adv. Exp. Med. Biol. 631 (2008) 40-53. doi:10.1007/978-0-387-78885-2_4.

[19] Y. Sun, T. Fukamachi, H. Saito, H. Kobayashi, ATP requirement for acidic resistance in Escherichia coli., J. Bacteriol. 193 (2011) 3072-3077. doi:10.1128/JB.00091-11. 
[20] K.J. Adolfsen, M.P. Brynildsen, Futile cycling increases sensitivity toward oxidative stress in Escherichia coli, Metab. Eng. 29 (2015) 26-35. doi:10.1016/j.ymben.2015.02.006.

[21] B.E. Cohen, Functional linkage between genes that regulate osmotic stress responses and multidrug resistance transporters: challenges and opportunities for antibiotic discovery., Antimicrob. Agents Chemother. 58 (2014) 640-646. doi:10.1128/AAC.02095-13.

[22] S.M. Ma, D.E. Garcia, A.M. Redding-Johanson, G.D. Friedland, R. Chan, T.S. Batth, et al., Optimization of a heterologous mevalonate pathway through the use of variant HMG-CoA reductases., Metab. Eng. 13 (2011) 588-597. doi:10.1016/j.ymben.2011.07.001.

[23] M.L. Barta, W.J. McWhorter, H.M. Miziorko, B. Geisbrecht, Structural basis for nucleotide binding and reaction catalysis in mevalonate diphosphate decarboxylase, Biochemistry. 51 (2012) 5611-5621. doi:10.1021/bi300591x.

[24] J.C. Vannice, D.A. Skaff, A. Keightley, J.K. Addo, G.J. Wyckoff, H.M. Miziorko, Identification in Haloferax volcanii of phosphomevalonate decarboxylase and isopentenyl phosphate kinase as catalysts of the terminal enzyme reactions in an archaeal alternate mevalonate pathway, J. Bacteriol. 196 (2014) 1055-1063. doi:10.1128/JB.01230-13.

[25] T. Baba, T. Ara, M. Hasegawa, Y. Takai, Y. Okumura, M. Baba, et al., Construction of Escherichia coli K-12 in-frame, single-gene knockout mutants: the Keio collection., Mol. Syst. Biol. 2 (2006) 2006.0008. doi:10.1038/msb4100050.

[26] T.S. Lee, R.A. Krupa, F. Zhang, M. Hajimorad, W.J. Holtz, N. Prasad, et al., 
BglBrick vectors and datasheets: A synthetic biology platform for gene expression., J. Biol. Eng. 5 (2011) 12. doi:10.1186/1754-1611-5-12.

[27] J. Degenhardt, T.G. Köllner, J. Gershenzon, Monoterpene and sesquiterpene synthases and the origin of terpene skeletal diversity in plants., Phytochemistry. 70 (2009) 1621-1637. doi:10.1016/j.phytochem.2009.07.030.

[28] J. Alonso-Gutierrez, R. Chan, T.S. Batth, P.D. Adams, J.D. Keasling, C.J. Petzold, et al., Metabolic engineering of Escherichia coli for limonene and perillyl alcohol production, Metab. Eng. 19 (2013) 33-41. doi:10.1016/j.ymben.2013.05.004.

[29] E.M. Kim, J.H. Eom, Y. Um, Y. Kim, H.M. Woo, Microbial Synthesis of Myrcene by Metabolically Engineered Escherichia coli., J. Agric. Food Chem. 63 (2015) 4606-4612. doi:10.1021/acs.jafc.5b01334.

[30] C. Wang, S.H. Yoon, A.A. Shah, Y.R. Chung, J.Y. Kim, E.S. Choi, et al., Farnesol production from Escherichia coli by harnessing the exogenous mevalonate pathway., Biotechnol. Bioeng. 107 (2010) 421-9. doi:10.1002/bit.22831.

[31] D.S. Gogerty, T.A. Bobik, Formation of isobutene from 3-hydroxy-3methylbutyrate by diphosphomevalonate decarboxylase., Appl. Environ. Microbiol. 76 (2010) 8004-8010. doi:10.1128/AEM.01917-10.

[32] D. Krepkiy, H.M. Miziorko, Identification of active site residues in mevalonate diphosphate decarboxylase: implications for a family of phosphotransferases., Protein Sci. 13 (2004) 1875-1881. doi:10.1110/ps.04725204.

[33] S. Wagner, M.M. Klepsch, S. Schlegel, A. Appel, R. Draheim, M. Tarry, et al., 
Tuning Escherichia coli for membrane protein overexpression., Proc. Natl. Acad. Sci. USA. 105 (2008) 14371-14376. doi:10.1073/pnas.0804090105.

[34] J.B. Bonanno, C. Edo, N. Eswar, U. Pieper, M.J. Romanowski, V. Ilyin, et al., Structural genomics of enzymes involved in sterol/isoprenoid biosynthesis., Proc. Natl. Acad. Sci. USA. 98 (2001) 12896-12901. doi:10.1073/pnas.181466998.

[35] D.S. Clark, H.W. Blanch, Biochemical Engineering, Second Edition, CRC Press, 1997. https://books.google.com/books?id=ST_p2AOApZsC\&pgis=1 (accessed August 12, 2015). 


\section{Figure legends}

Fig. 1. Original and two modified mevalonate pathways for isopentenol production. The original mevalonate pathway (pathway O) produces isopentenyl diphosphate (IPP), which is dephosphorylated by NudB, as an intermediate. Two modified pathways were proposed in this study: direct decarboxylation of mevalonate (pathway I) or decarboxylation of mevalonate diphosphate (pathway II) followed by de-phosphorylation of isopentenyl monophosphate (IP). Numbers of ATP and enzymes required for each pathway are summarized in the table. Ac-CoA, acetyl-CoA; AAc-CoA, acetoacetylCoA; HMG-CoA, 3-hydroxy-3-methyl-glutaryl-CoA; PMK, phosphomevalonate kinase; PMD, phosphomevalonate decarboxylase)

Fig. 2. GC/MS chromatogram (left) and mass spectra (right) of ethyl acetate-extracted metabolites detected from (A) control strain with three genes (ARK3a; atoB, HMGS, $H M G R$ ) and (B) engineered strains with four genes (ARK3b; atoB, HMGS, HMGR and $\left.P M D_{s c}\right)$. The mass spectrum of the peak that eluted at 9.49 min detected in ARK $3 \mathrm{~b}(\mathrm{~B})$ is very similar to that of the isopentenol standard (C), and is not present in the ethyl acetate blank (D). Arrows indicate masses of the peak at retention time of 9.49 min detected from both standard (C) and the engineered strain (B; ARK3b).

Fig. 3. Hydrolysis of IP and IPP by purified NudB or E. coli cell lysates. (A) IP hydrolysis. IP was hydrolyzed to isopentenol by $E$. coli cell lysates while isopentenol was not detected $(*)$ from other two reactions with or without purified NudB. (B) Profile of IP and IPP concentrations in in vitro hydrolysis reactions of IPP by purified NudB. 
Fig. 4. Identification of endogenous phosphatases for IP. (A) Isopentenol concentration $(\mu \mathrm{M})$ in the cell lysates of monophosphatase mutants. A total of 36 mutants (diamonds) —including $\triangle a g p$ (solid circle), $\triangle y q a B$ (solid triangle), $\triangle a p h A$ (solid square) mutants and wild type BW25113 (open circle)—were screened. The grey line represents the average $(251.9 \mu \mathrm{M})$ of isopentenol concentrations detected from all mutants. (B) Isopentenol converted from $1 \mathrm{mM}$ IP by cell lysates of wild type (BW25113, empty circle), $\triangle a g p$ (circle), $\triangle y q a B$ (triangle) and $\triangle a p h A$ (square) mutants. (C) Isopentenol converted from $500 \mu \mathrm{M}$ IP by cell lysates of wild type (solid lines) or each mutant (dotted lines) with overexpression of the corresponding gene: agp (circle), yqaB (triangle) and $\operatorname{aph} A$ (square).

Fig. 5. Effect of $a p h A$ expression on isopentenol production in pathway II. Isopentenol from pathway II with (dark grey bar) or without aphA expression (white bar). Optical density of cell cultures at $600 \mathrm{~nm}\left(\mathrm{OD}_{600}\right)$ for pathway II with (solid circle) or without aphA expression (open circle)

Fig. 6. Effect of mutations on isopentenol production with IPP-bypass pathway II. (A) location of $\mathrm{R} 74$ and $\mathrm{I} 145$ in $\mathrm{PMD}_{\text {sc. }}$ Blue meshes are essential residues for catalysis and substrate binding, and pink meshes are residues selected for mutagenesis. Electrostatic interactions were not clearly found between substrates and these residues and the distance between phosphate group of the substrate analog (6-fluoromevalonate 5-diphosphate (FMVAPP)) and R74 or I145 residue were $12.5 \AA$ or $15.0 \AA$, respectively. (B) Curve fittings and kinetics of $\mathrm{PMD}_{\mathrm{sc}}$ wild type and four mutants (K22M, R74H, I145F and 
T209D). (C) Isopentenol production from strains with pathway II containing different PMD mutants including wild type (WT, black square), R74H (open circle), I145F (grey circle) and R74H/I145F double mutants (black circle).

Fig. 7. Effect of different "top" portions on isopentenol production in E. coli with pathway O or with pathway II. Four different "top" portions have different HMGS and HMGR sequences, which are original sequences from. S. cerevisiae (MevTo), codonoptimized sequences of S. cerevisiae (MevTco), sequences from S. aureus (MTSA) and sequences from D. acidovorans (MTDA). Isopentenol production was measured at 24 hours (white dotted bar) and at 48 hours (grey bars).

Fig. 8. IPP toxicity in Pathway O. (A) Growth of four strains containing Pathway O without (black, square) or with expression of additional MK (white square); Pathway II without (black, circle) or with expression of additional MK (white circle). Isopentenol production from four strains containing Pathway $\mathrm{O}$ without or with expression of additional MK; Pathway II without or with expression of additional MK.

Fig. 9. Effect of reduced aeration conditions on isopentenol production in E. coli. (A) Isopentenol titers (per $\mathrm{OD}_{600}$ ) of two strains with pathway $\mathrm{O}$ or with pathway II under higher (200 rpm) or lower (30 rpm) aeration conditions. (B) Relative isopentenol titers (total) of two pathways under lower aeration conditions (30 rpm) compared to those under higher aeration conditions (200 rpm). 
Table 1 List of strains and plasmids used in this study

\begin{tabular}{|c|c|c|}
\hline Strains & Description & Reference \\
\hline$\triangle a p h A$ & E. coli $\mathrm{K} 12 \mathrm{BW} 25113$ AaphA & Keio Collection [25] \\
\hline$\triangle a g p$ & E. coli $\mathrm{K} 12 \mathrm{BW} 25113 \triangle a g p$ & Keio Collection [25] \\
\hline$\triangle y q a B$ & E. coli $\mathrm{K} 12 \mathrm{BW} 25113 \triangle y q a B$ & Keio Collection [25] \\
\hline ARK1a & JBEI-12056 + JBEI-9348 & This study \\
\hline ARK1b & JBEI-6824 + JBEI-9348 & This study \\
\hline ARK1c & JBEI-6831 + JBEI-9348 & This study \\
\hline ARK1d & JBEI-7575 + JBEI-9348 & This study \\
\hline ARK1e & JBEI-6818 + JBEI-6833 & This study \\
\hline ARK1f & JBEI-6818 + JBEI-6834 & This study \\
\hline ARK2a & JBEI-9310 + JBEI-9314 & This study \\
\hline ARK2b & JBEI-9309 + JBEI-9314 & This study \\
\hline ARK2c & JBEI-9312 + JBEI-9314 & This study \\
\hline ARK2d & JBEI-9311 + JBEI-9314 & This study \\
\hline ARK2e & JBEI-12051 + JBEI-9314 & This study \\
\hline ARK2f & JBEI-12051 + JBEI-12064 & This study \\
\hline ARK2aa & JBEI-12050 + JBEI-9314 & This study \\
\hline ARK2 $a_{M 1}$ & JBEI-9310 + JBEI-12060 & This study \\
\hline $\mathrm{ARK} 2 \mathrm{a}_{\mathrm{M} 2}$ & JBEI-9310 + JBEI-12061 & This study \\
\hline ARK2 $\mathrm{a}_{\mathrm{M} 3}$ & JBEI-9310 + JBEI-12062 & This study \\
\hline ARK3a & JBEI-3100 + JBEI-12229 & This study \\
\hline ARK3b & JBEI-3100 + JBEI-3277 & This study \\
\hline ARK4 & JBEI-9310 + JBEI-12054 & This study \\
\hline ARK5 & JBEI-9310 + JBEI-12059 & This study \\
\hline Plasmids & Description & Reference \\
\hline JBEI-6818 & pBbA5c-MevTo-MKсо-РМКсо & {$[6]$} \\
\hline JBEI-6824 & pBbA5c-MevTco-BBa1002-pTrc-MKco-PMKсо & {$[6]$} \\
\hline JBEI-6831 & pBbA5c-MTSA-BBa1002-pTrc-MKco-PMKco & {$[6]$} \\
\hline JBEI-6833 & pTrc99a-NudB-PMDsc & [6] \\
\hline JBEI-6834 & pTrc99a-NudB-PMDsc-Mkco & [6] \\
\hline JBEI-7575 & pBbA5c-MTDA-BBa1002-pTrc-MKco-PMKco & Gift from Eunmi Kim \\
\hline JBEI-9309 & pBbA5c-MevTco-BBa1002-pTrc-MKco & This study \\
\hline JBEI-9310 & pBbA5c-MevTo-BBa1002-pTrc-MKco & This study \\
\hline JBEI-9311 & pBbA5c-MTDA-BBa1002-pTrc-MKco & This study \\
\hline JBEI-9312 & pBbA5c-MTSA-BBa1002-pTrc-MKco & This study \\
\hline JBEI-9314 & pTrc99a-PMDsc & This study \\
\hline JBEI-9348 & pTrc99a-PMDsc-NudB & This study \\
\hline JBEI-12050 & pBbA5c-MevTo-BBa1002-pTrc-MKco-aphA & This study \\
\hline JBEI-12051 & pBbA5c-MevTo-MKсо & This study \\
\hline
\end{tabular}




\begin{tabular}{lll} 
JBEI-12052 & pSKB3-PMDsc & This study \\
JBEI-12053 & pSKB3-PMDsc_K22M & This study \\
JBEI-12054 & pTrc99a-PMDse & This study \\
JBEI-12055 & pSKB3-PMDsc_T209D & This study \\
JBEI-12056 & pBbA5c-MevTo-BBa1002-pTrc-MKco-PMKco & This study \\
JBEI-12057 & pSKB3-PMDsc_R74H & This study \\
JBEI-12058 & pSKB3-PMDsc_I145F & This study \\
JBEI-12059 & pTrc99a-PMDhv & This study \\
JBEI-12060 & pTrc99a-PMDsc_R74H & This study \\
JBEI-12061 & pTrc99a-PMDsc_I145F & This study \\
JBEI-12062 & pTrc99a-PMDsc_R74H/I145F & This study \\
JBEI-12064 & pTrc99a-PMDsc-MKco & This study \\
JBEI-12229 & pE1a-PMDsc & This study \\
\hline
\end{tabular}


Table 2. Kinetic parameters of PMD wild type, $\mathrm{PMD}_{\mathrm{sc}}$ mutants, $\mathrm{PMD}_{\mathrm{se}}, \mathrm{PMD}_{\mathrm{hv}}$ from other literatures.

\begin{tabular}{lccccccc}
\hline \multicolumn{2}{c}{ Name } & $\begin{array}{c}\mathrm{K}_{\mathrm{m}} \\
(\mathrm{mM})\end{array}$ & $\begin{array}{c}\mathrm{k}_{\text {cat }} \\
\left(\mathrm{s}^{-1}\right)\end{array}$ & $\begin{array}{c}\mathrm{k}_{\mathrm{cat}} / \mathrm{K}_{\mathrm{m}} \\
\left(\mathrm{s}^{-1} \mathrm{M}^{-1}\right)\end{array}$ & \% of WT & Substrate & Reference \\
\hline PMD $_{\text {sc }}$ & $\mathrm{WT}$ & 0.99 & 0.14 & $1.4 \times 10^{2}$ & $100 \%$ & & \\
& $\mathrm{R} 74 \mathrm{H}$ & 0.77 & 0.23 & $3.0 \times 10^{2}$ & $220 \%$ & & \\
& $\mathrm{~K} 22 \mathrm{M}$ & 2.47 & 0.09 & $3.5 \times 10^{1}$ & $25 \%$ & MVAP & This study \\
& $\mathrm{T} 209 \mathrm{D}$ & 0.99 & 0.13 & $1.3 \times 10^{2}$ & $98 \%$ & & \\
& $\mathrm{I} 145 \mathrm{~F}$ & 1.36 & 0.28 & $2.0 \times 10^{2}$ & $147 \%$ & & MVAP \\
\hline PMD $_{\mathrm{hv}}$ & & 0.159 & 3.5 & $2.2 \times 10^{4}$ & & MVAPP & {$[23]$} \\
PMD $_{\text {se }}$ & 0.009 & 5.9 & $6.5 \times 10^{5}$ & & MVAPP & {$[32]$} \\
PMD $_{\text {sc }}$ & 0.123 & 5.4 & $4.0 \times 10^{4}$ & & & \\
\hline
\end{tabular}




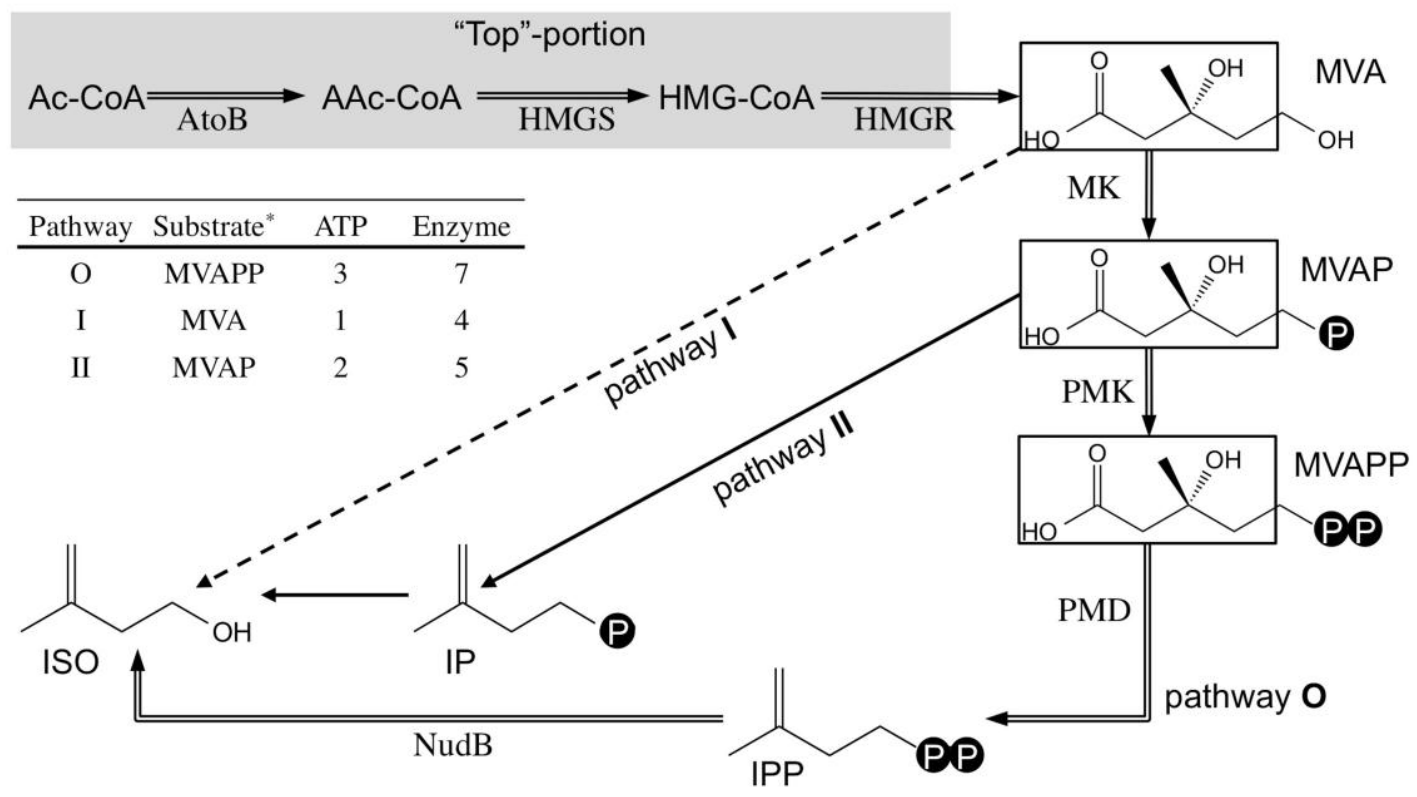


(A)

(B)
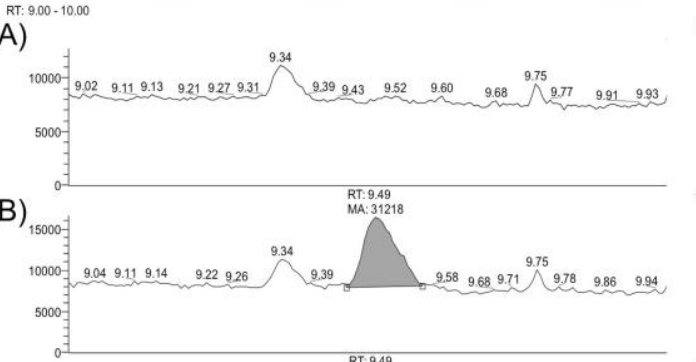

(C)

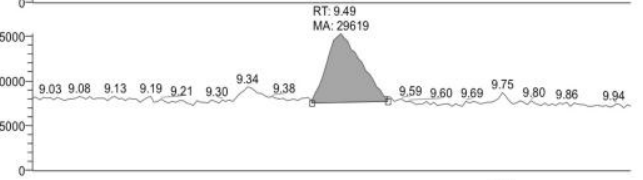

(D)
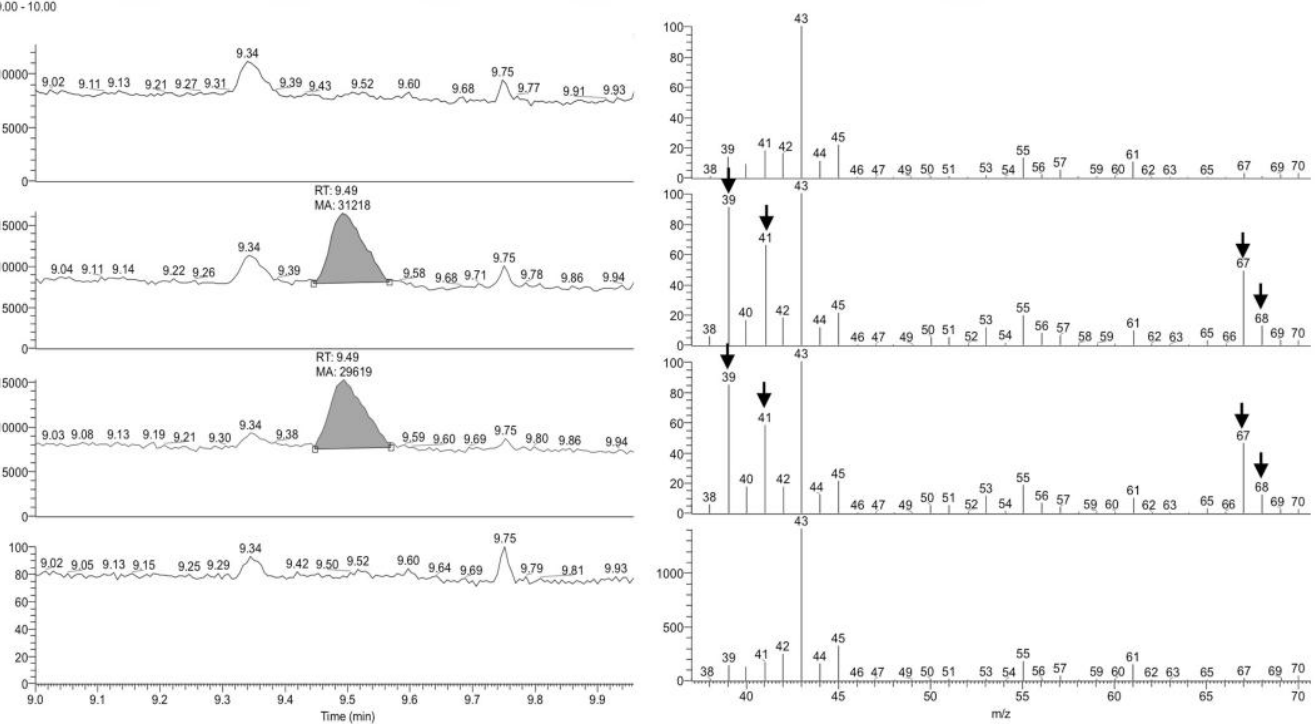
(A)
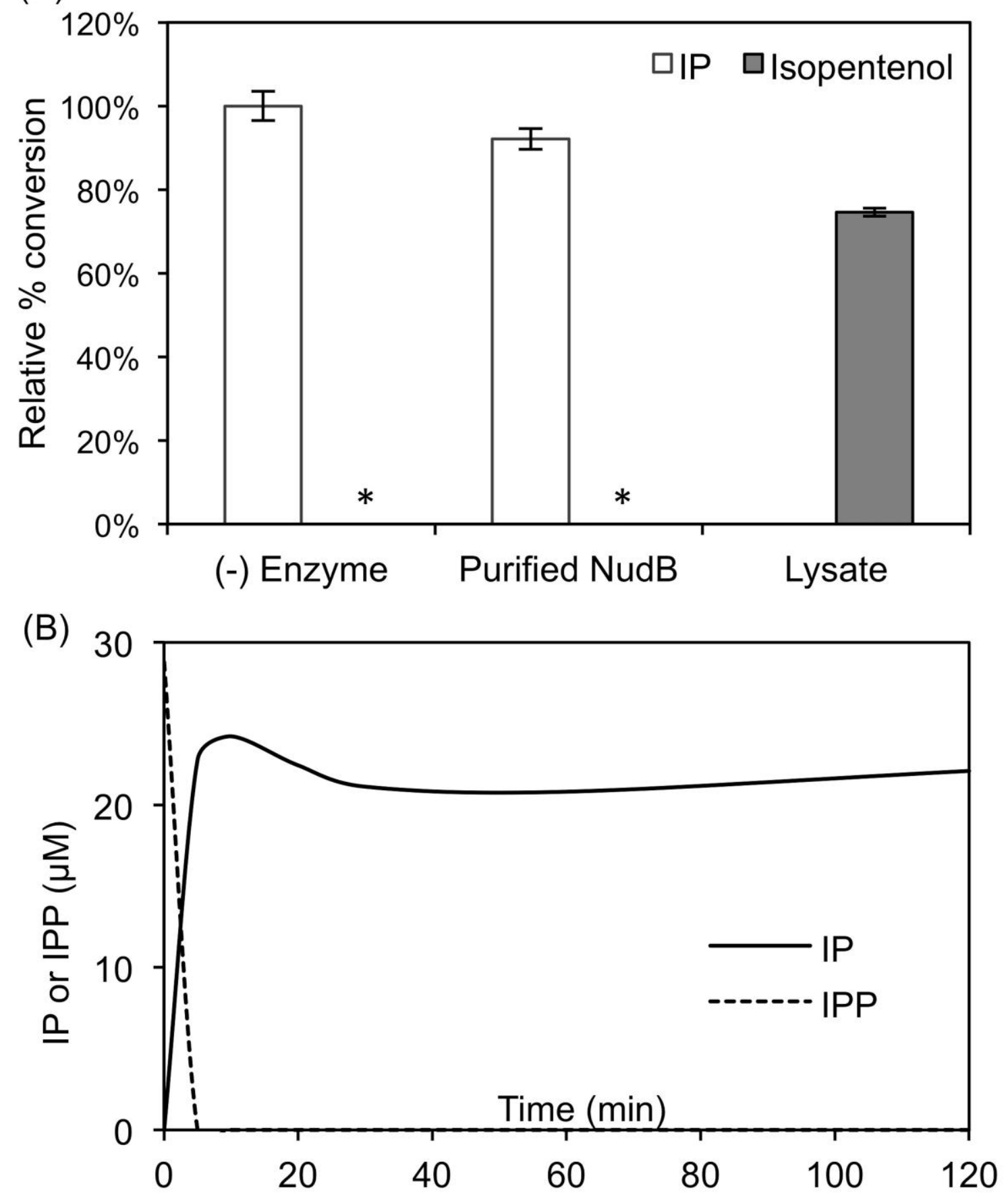

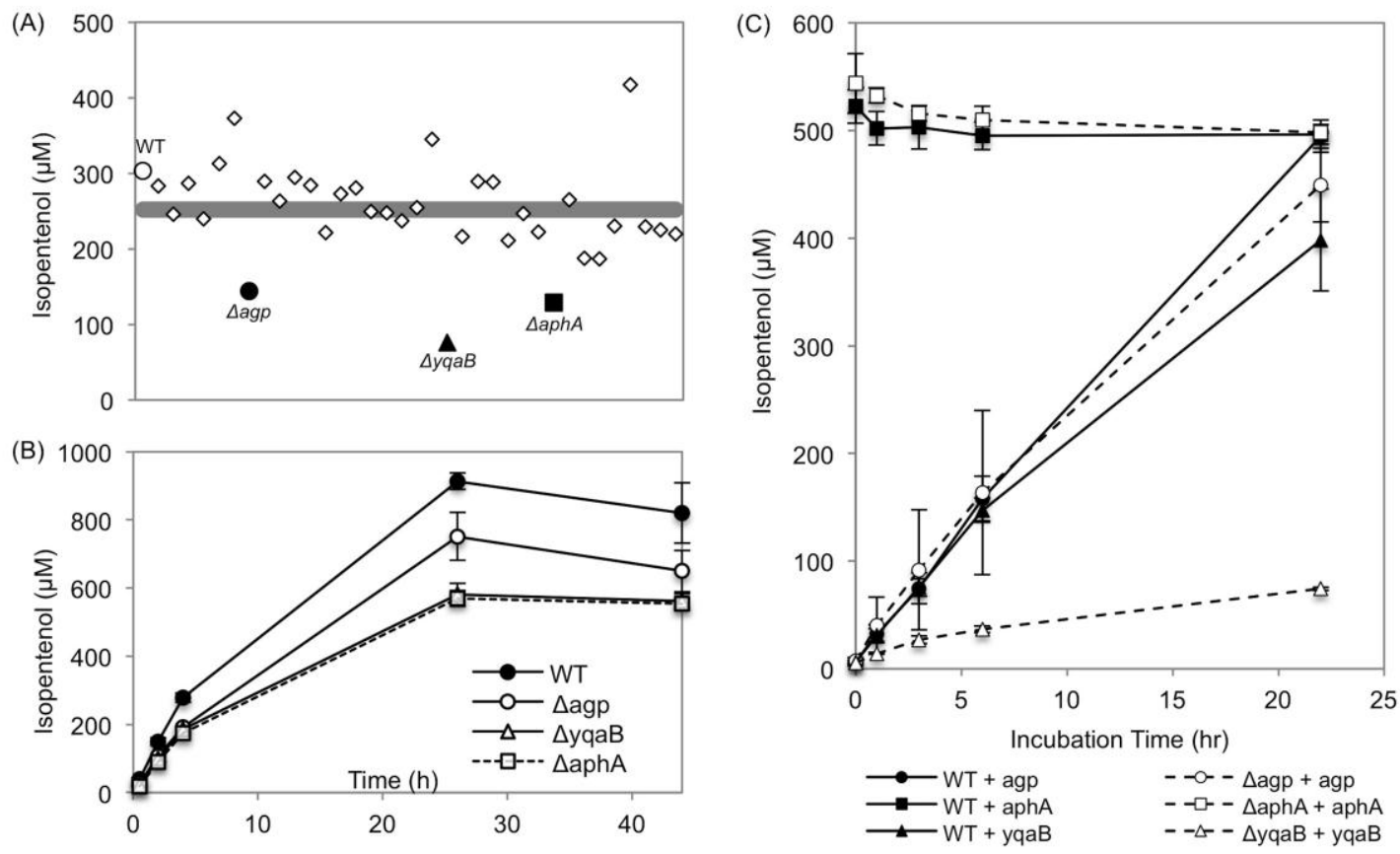


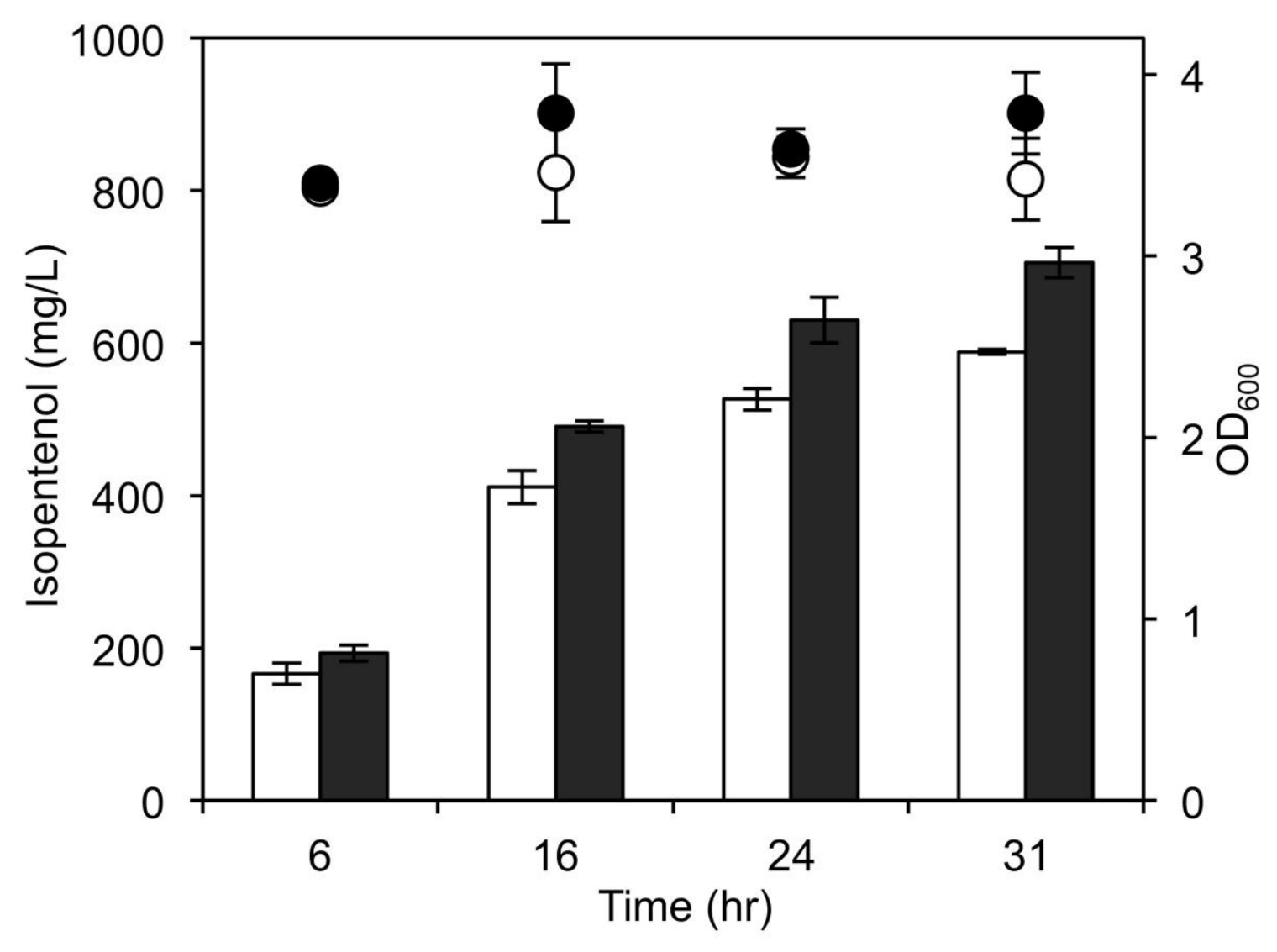

$\square$ Isopentenol, ARK2a (pathway II)

- Isopentenol, ARK2aa (pathway II + aphA)

$\circ \mathrm{OD}_{600}$, ARK2a (pathway II)

- $\mathrm{OD}_{600}$, ARK2aa (pathway II + aphA) 
(A)

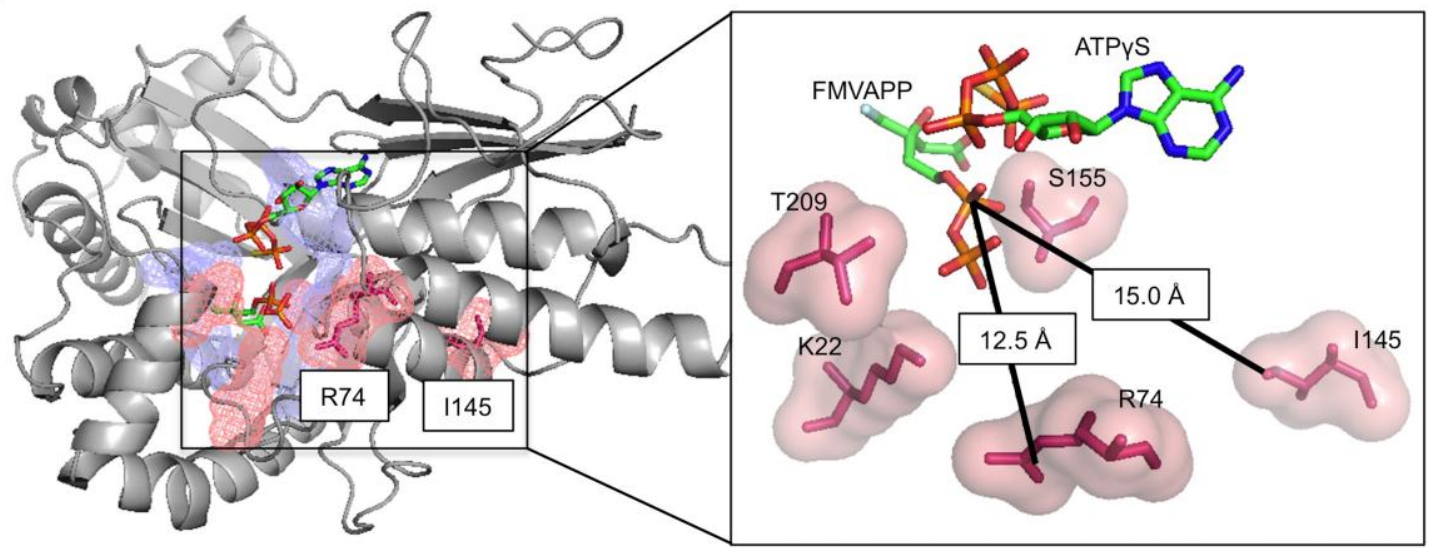

(B)

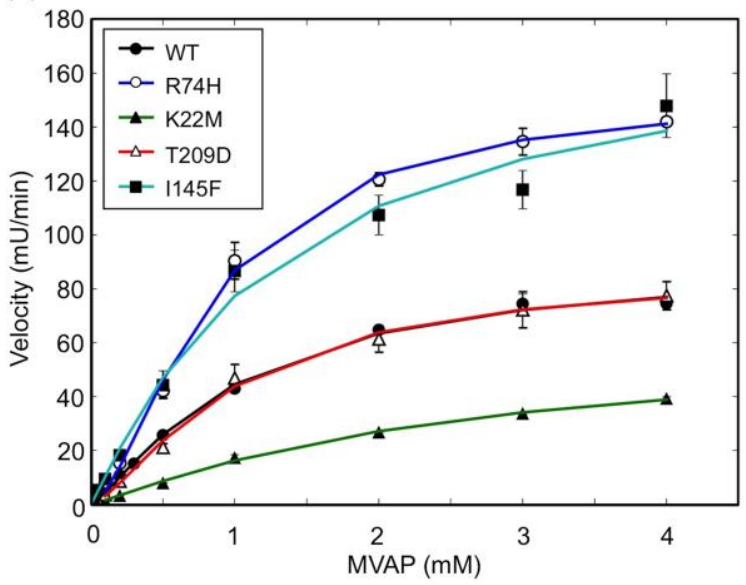

(C)

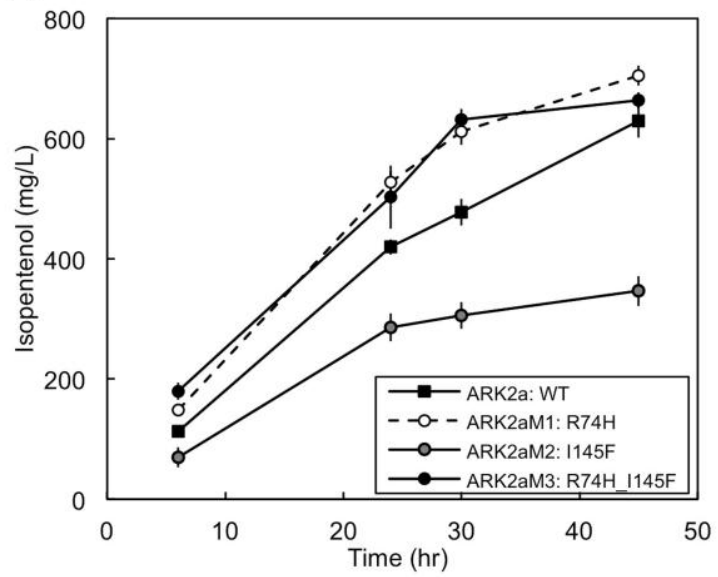




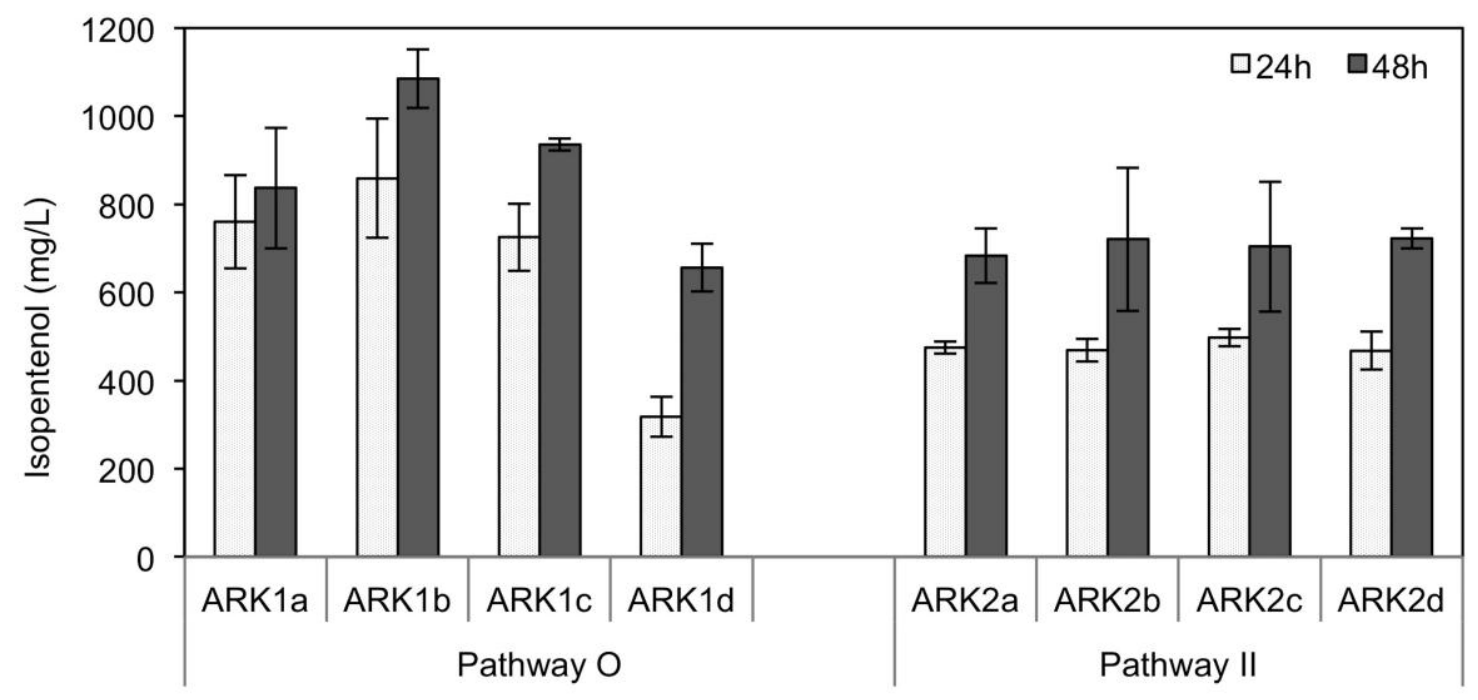

"top" portions: $\mathbf{a}=$ MevTo, $\mathbf{b}=$ MevTco, $\mathbf{c}=$ MTSA, $\mathbf{d}=$ MTDA 

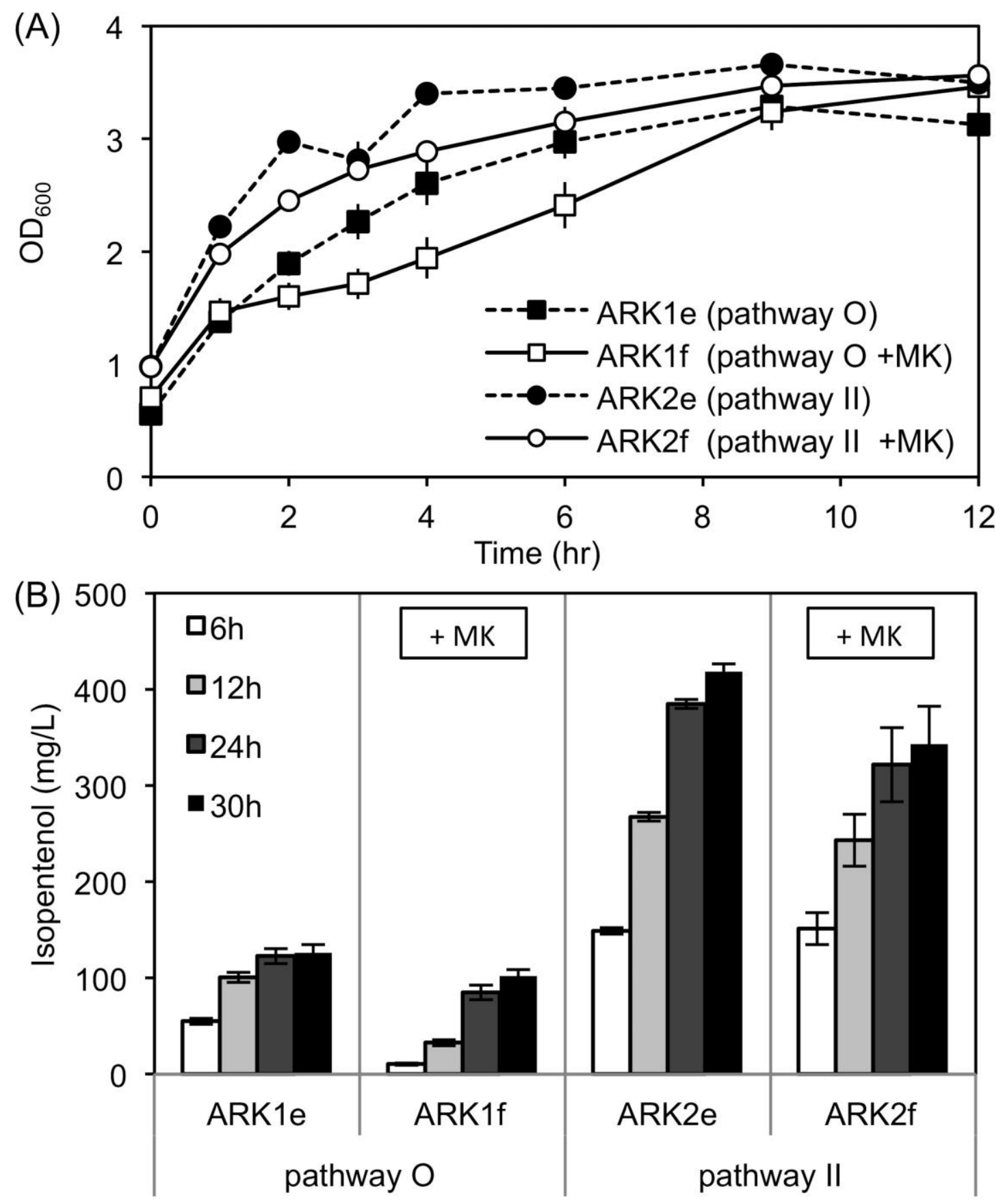


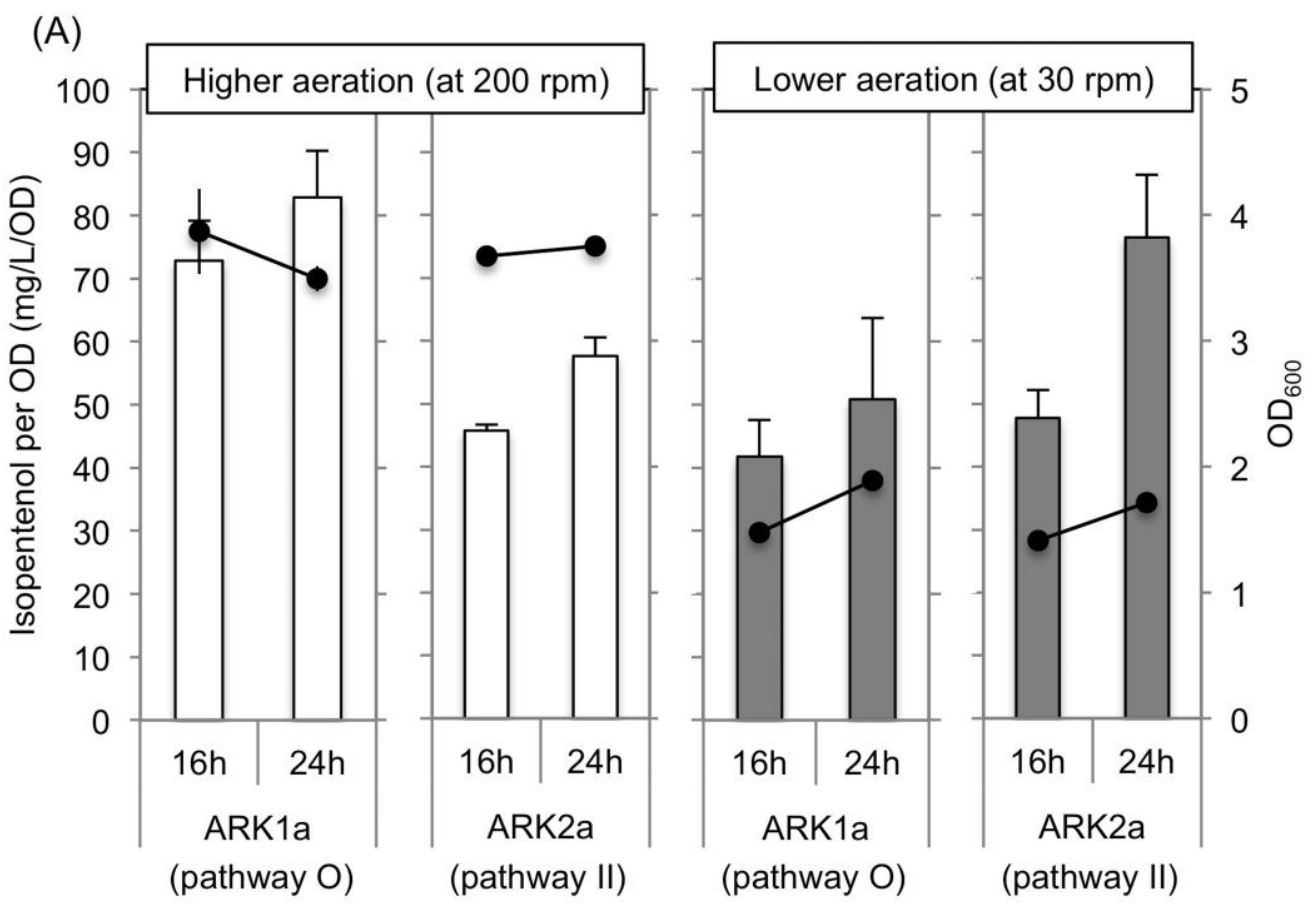

(B)

\begin{tabular}{ll|cc}
\hline & & $16 \mathrm{~h}$ & $24 \mathrm{~h}$ \\
\cline { 2 - 4 }$\frac{}{\text { Isopentenol titers at 30 rpm }} \times 100$ & ARK1a (pathway O) & $22 \%$ & $33 \%$ \\
\hline Isopentenol titers at 200 rpm & ARK2a (pathway II) & $40 \%$ & $60 \%$ \\
\hline
\end{tabular}

